\title{
High pressure chiral recognition experiments - a route to homochiral 4-fluorocyclohexenones
}

\author{
Daniel Meibom, ${ }^{* a}$ Hilmar Weinmann, ${ }^{b}$ and Ekkehard Winterfeldt ${ }^{\mathrm{c}}$
}

${ }^{a}$ Bayer AG, Pharma Research, D-42096 Wuppertal, and ${ }^{b}$ Schering AG, Müllerstr. 178, 13353 Berlin, Germany, and ${ }^{c}$ Institut für Organische Chemie der Universität Hannover, Schneiderberg 1b, 30167 Hannover, Germany

E-mail: winterfeldt@mbox.oci.uni-hannover.de

This paper is dedicated to Professor Binne Zwanenburg - a great colleague and a good friend - on the occasion of his $70^{\text {th }}$ birthday

(received 08 Jun 03; accepted 21 Oct 03; published on the web 27 Oct 03)

\begin{abstract}
4-Fluorocyclohexadienones, prepared by an improved Jacquesy-process, were shown to undergo highly face-selective Diels-Alder reactions with the homochiral cyclopentadiene 1. Some of the diastereoselective transformations, quite unique rearrangements of $\alpha$-hydroxy compounds resulting from nucleophilic attack to the carbonyl group as well as selected Retro-Diels-Alder reactions, are reported.
\end{abstract}

Keywords: Asymmetric synthesis, chiral recognition, cycloadditions, high pressure chemistry, fluorocompounds

\section{Introduction}

In recent papers, we communicated the potential of the homochiral cyclopentadiene $\mathbf{1}^{1}$ for sequential diastereoselective cycloadditions, ${ }^{2}, 3$ kinetic resolution ${ }^{4}$ and differentiation of enantiotopic double bonds. ${ }^{4,5,7}$ In the latter case, cycloadditions with cyclohexadienones of type 2 were shown to follow the very obvious pathway which places the small residue "S" into the $\alpha$-endo-position (Scheme 1).

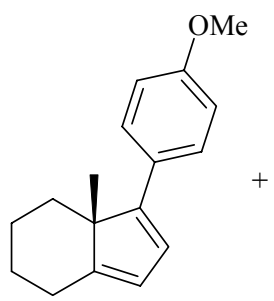

1

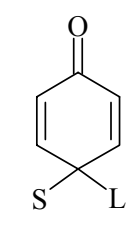

2

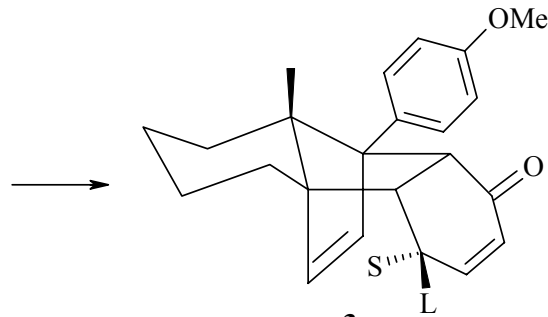

3

\section{Scheme 1}


With various disubstituted cyclohexadienones as well as spiro-cyclohexadienones this was shown to be a highly selective and absolutely reliable transformation, ${ }^{7}$ which however, due to immediate formation of phenols, cannot be exercised with $\mathrm{S}=\mathrm{H}$.

In such a situation with a need for a non-enolizable hydrogen replacement with comparable space demand the fluorine is the candidate of choice. ${ }^{8}$ The special influence of this electron attracting substituent on cycloaddition rates $^{7}$ and biological activity, ${ }^{9,10,11}$ that has been reported for various cyclohexenone- and spiro-cyclohexenone derivatives, ${ }^{12}$ certainly enhances the motivation for these investigations.

\section{Results and Discussion}

\section{Preparation of dienophiles}

Looking for a simple and broadly applicable route to fluorinated cyclohexadienones of type 5, we selected the method published by Jacquesy et. al. ${ }^{13}$ employing bis-trifluoroacetoxy iodobenzene (PIFA) as oxidant and pyridinium poly-hydrogenfluoride (PPHF) as nucleophile. The reaction probably proceeds through intermediate 4 (Scheme 2).

Unfortunately, in our hands even the quite simple methyl derivative 5a could only be obtained in $34 \%$. In addition to low yields and bad reproduceability we also observed the formation of phenol dimers and of typically dark brown polymers formed in phenol oxidations. ${ }^{14}$ We therefore in some cases chose bis-acetoxy iodobenzene (PIDA) as the weaker oxidant and inverse addition in order to maintain a low stationary phenol concentration (see experimental). Under these conditions, we consistently obtained acceptable yields for 5a, 5c, 5e and $\mathbf{7}$ together with limited polymer formation.
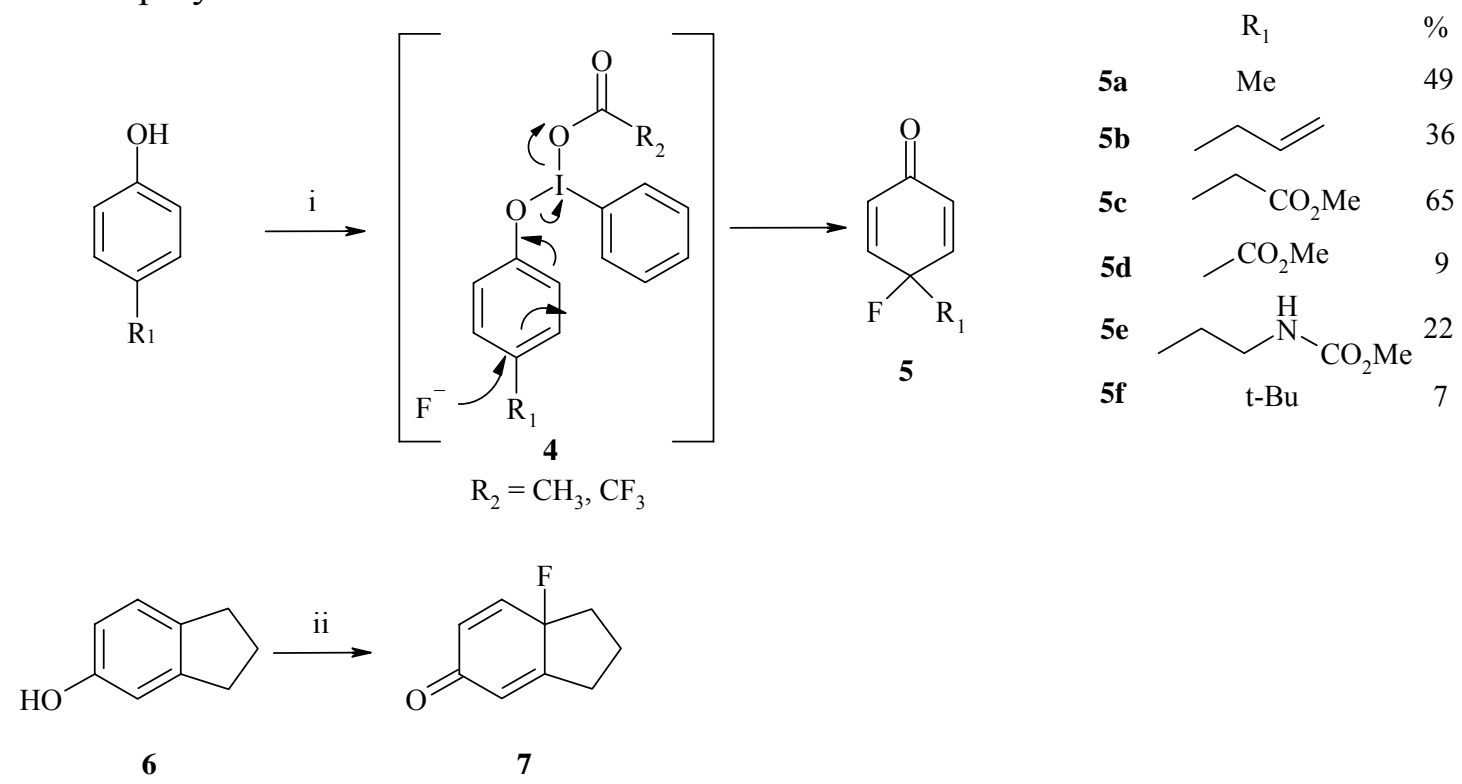

Scheme 2. $\mathrm{i}$ : $\mathrm{Ar}-\mathrm{I}(\mathrm{OR})_{2}$, PPHF, $\mathrm{CH}_{2} \mathrm{Cl}_{2}$; ii: PIDA, PPHF, $\mathrm{CH}_{2} \mathrm{Cl}_{2}, 0^{\circ} \mathrm{C}, 55 \mathrm{~min}, 47 \%$. 
Since we deal with simple starting materials the optimization process was stopped at this stage. It should be noted that with these modifications even the bicyclic fluoride 7 and the tertbutyl derivative $\mathbf{5 f}$ are accessible, however, in the case of $5 \mathbf{f}$ only in $7 \%$ yield. These data clearly demonstrate that the presence of an acceptor group (see 5d) as well as space demanding substituents (5e, 5f) lower the yield of the transformation.

As expected, all these cyclohexadienones (5a-5f) suffer from air oxidation and photodegradation. However, they can be kept for months if they are stored in the refrigerator at $0{ }^{\circ} \mathrm{C}$ under argon.

\section{Cycloaddition experiments}

With a good selection of dienophiles available, we focused on a detailed investigation of their cycloaddition behaviour. In order to demonstrate the rate enhancing effects of the fluoro atom ${ }^{7}$ we started with a high pressure competition experiment employing methyl derivative 5a, which had already been shown to undergo high yielding, diastereoselective cycloadditions at $6,5 \mathrm{kbar}^{5}$ and the corresponding quinol 8 (Scheme 3).

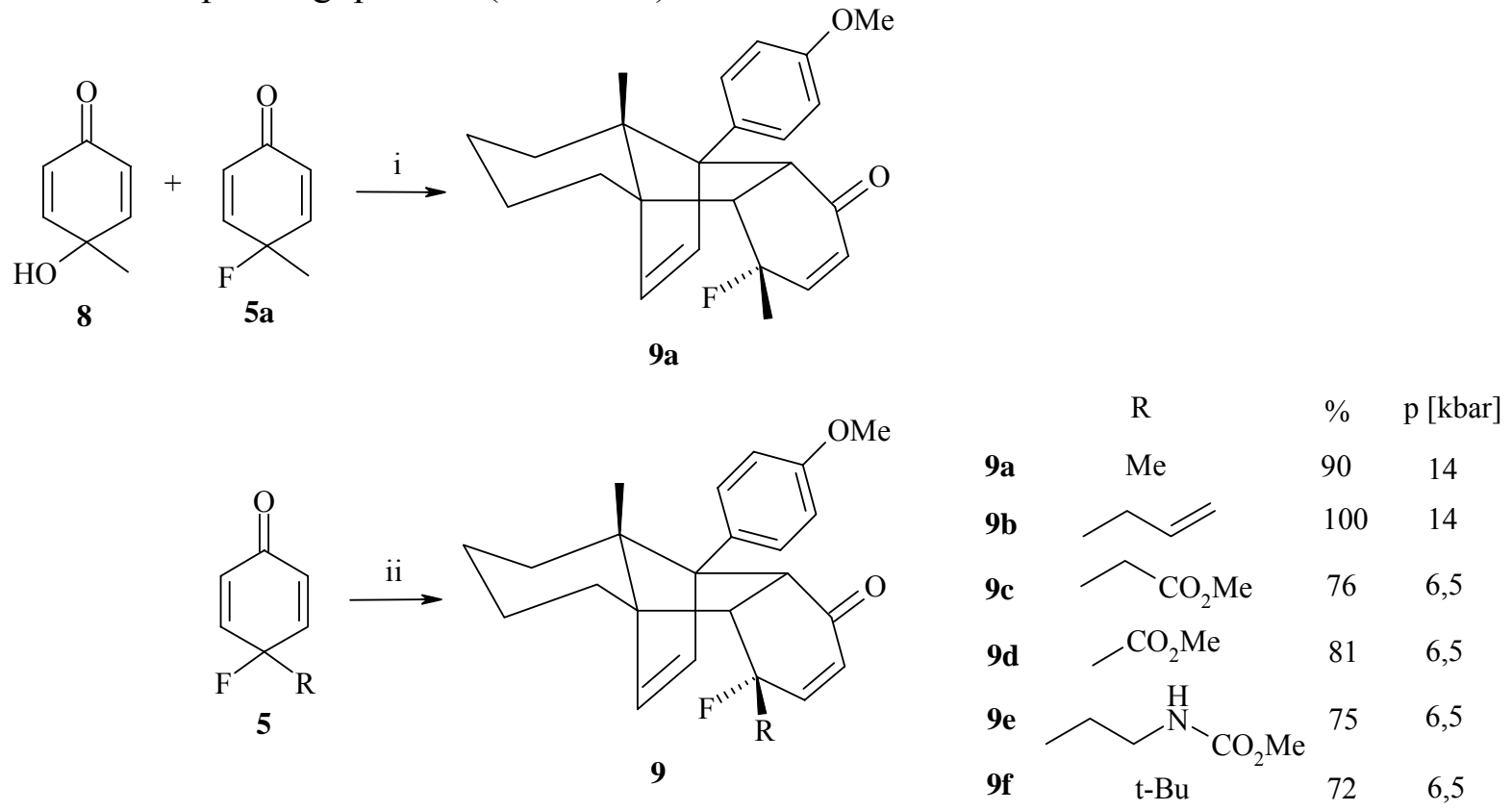

Scheme 3. i: 1, 14 kbar, RT, 6 d; ii: 1, 6.5-14 kbar, $\mathrm{CH}_{2} \mathrm{Cl}_{2}, \mathrm{RT}, 7-14 \mathrm{~d}$.

Although this competition experiment was run at $14 \mathrm{kbar}$ for six days, only the fluoro derivative 9a was formed and proved to be identical to the product obtained earlier at 6,5 kbar. Therefore the cycloadditions with $\mathbf{5 b} \mathbf{b} \mathbf{f}$ could be run at both pressures and both cases smoothly provided the corresponding adducts $\mathbf{9 b - 9 f}$ in good to excellent yields.

A special observation that was particularly made starting from $5 \mathbf{b}$ and $\mathbf{5 c}$ deserves further comments. In some cases without any detectable reason or predictability, no cycloaddition took place at all and the whole material was completely destroyed leading to a black suspension. 
Assuming the formation of radicals to be responsible for these undesired processes, we tried a few radical scavengers and were finally successful with the addition of 4-N-acetylamino tetramethylpiperidin-N-oxyl (TEMPO), ${ }^{15}$ which leads to perfectly reproduceable yields for 9a, 9b and 9c as given in Scheme 3.

HPLC- as well as NMR-data proved all the cycloadducts to be single stereo- and regioisomers. Similarities to the well established data of $9 \mathbf{a}^{5}$ together with NOE measurements (see experimental) indicated the configuration as portrayed in Scheme 3.

Finally, we also studied the Diels-Alder chemistry of the two bicyclic dienophiles $\mathbf{7}$ and $\mathbf{1 1 .}$ While 7 resulted directly from the fluorination process (see Scheme 2), 11 was prepared from $5 \mathbf{e}$ via an intramolecular Michael addition (Scheme 4).
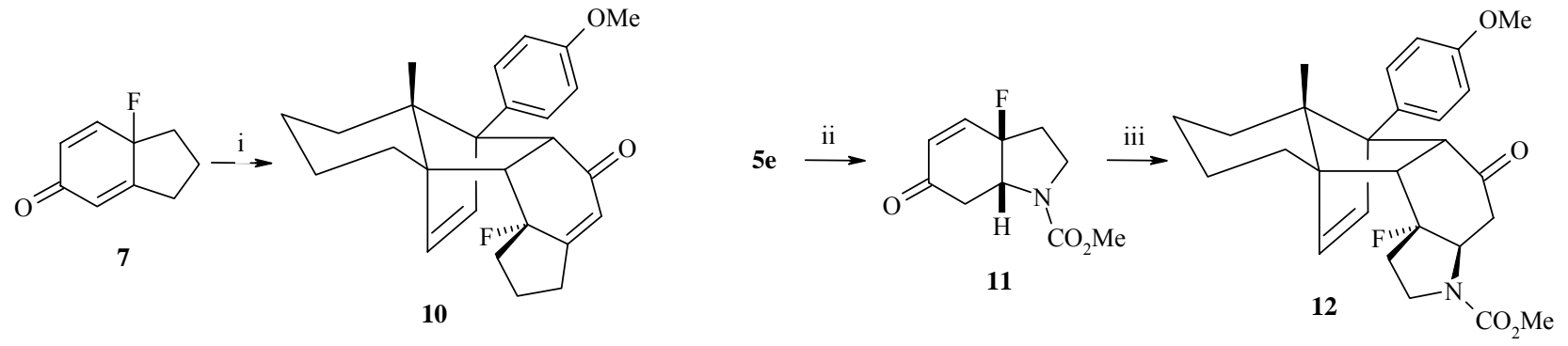

Scheme 4. i: 1, 6.5 kbar, $\mathrm{CH}_{2} \mathrm{Cl}_{2}$, RT, 9 d, 14\%; ii: $\mathrm{NaHCO}_{3}, \mathrm{H}_{2} \mathrm{O}, \mathrm{DMSO}$, overnight, 66 \%; iii: 1, 14 kbar, $\mathrm{CH}_{2} \mathrm{Cl}_{2}$, RT, $14 \mathrm{~d}, 36 \%$.

In both cases the yields at 6,5 kbar are low (starting from 11 only $4 \%$ cycloadduct). Therefore, we repeated this cycloaddition at $14 \mathrm{kbar}$ for a fortnight and obtained $36 \%$ of cycloadduct 12 instead. Taking into consideration that a kinetic resolution is undergone starting from the racemic mixture $\mathbf{1 1}$ the yield was clearly improved. The retro process with the material thus obtained provides excellent yields of enantiomerically pure 11, affording a simple route to homochiral fluorinated alkaloid building blocks.

\section{Selective transformations of cycloadducts}

It had been observed in numerous cases before that the special endo-configuration of adducts $\mathbf{9}$, 10 and 12 together with their conformational rigidity result in completely diastereoselective $\beta$ approach - in electrophilic as well as nucleophilic attack to the cyclohexenone moiety. ${ }^{4,5}$

For instance this could be shown for the "flash-dihydroxylation" followed by ketal formation and also for the chemo- and diastereoselective nucleophilic epoxidation ${ }^{17}$ of the allyl derivative $\mathbf{9 b}$ (Scheme 5). 


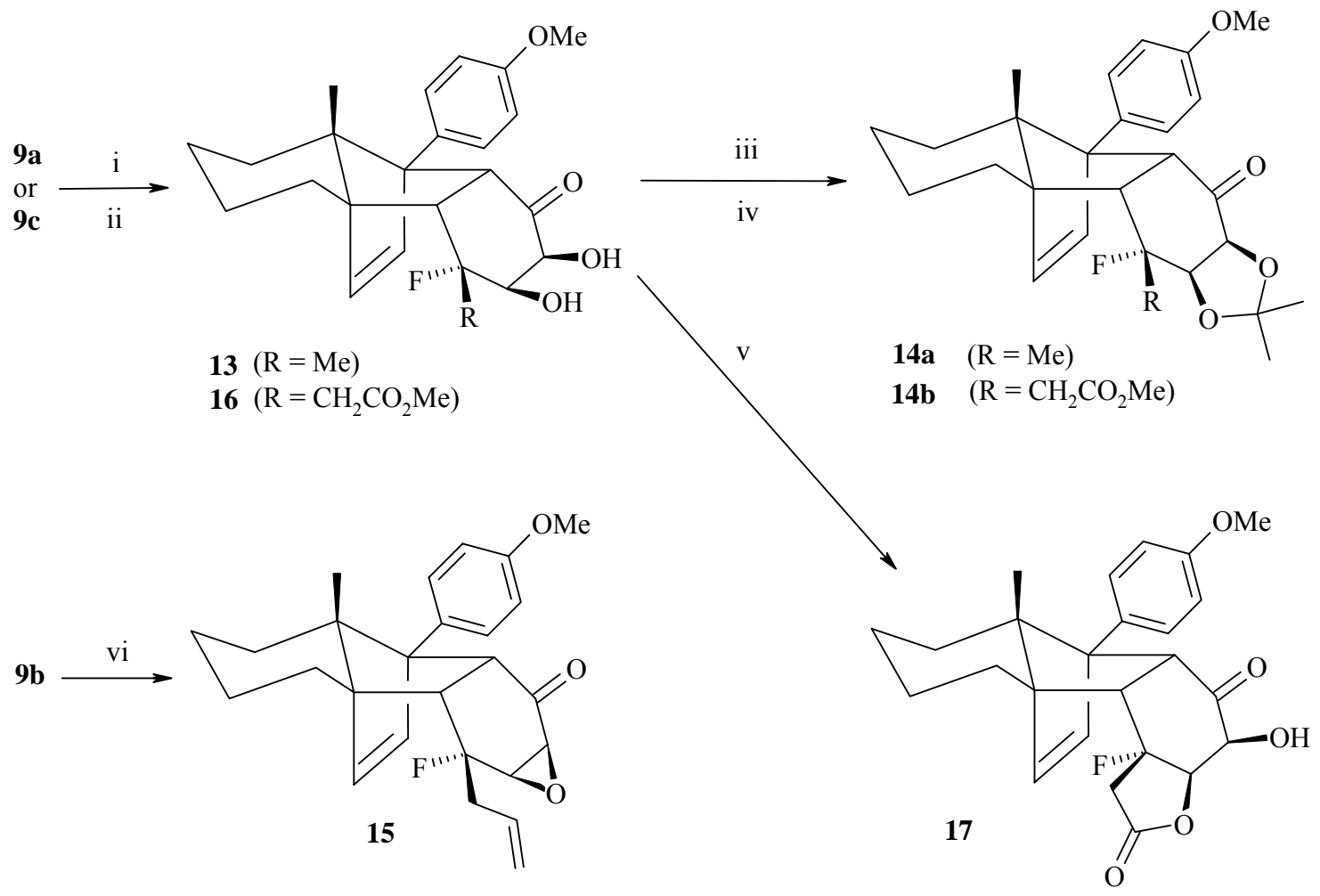

Scheme 5. i: 9a, $\mathrm{RuCl}_{3}, \mathrm{NaIO}_{4}$, EtOAc, $\mathrm{CH}_{3} \mathrm{CN}$, RT, 40 min, 71 \%; ii: 9c, $\mathrm{RuCl}_{3}, \mathrm{NaIO}_{4}, \mathrm{EtOAc}$ $\mathrm{CH}_{3} \mathrm{CN}$, RT, 40 min, $71 \%$; iii: 13, 2-methoxypropene, p-TsOH, DMF, RT, overnight, $99 \%$; iv: 16, 2,2-dimethoxypropane, p-TsOH, DMF, $100{ }^{\circ} \mathrm{C}, 1 \mathrm{~h}, 73$ \%; v: 16, HCl, THF, RT, 5 d, 22 \%; vi: $\mathrm{H}_{2} \mathrm{O}_{2}, \mathrm{NaOH}, \mathrm{THF}, \mathrm{MeOH}, \mathrm{RT}, 40 \mathrm{~min}, 97 \%$.

Interestingly, the ester derivative 9c gave different results when we tried to apply the flashhydroxylation - ketal formation sequence. Although hydroxylation worked as expected, ketalisation, which leads to nearly quantitative yields with methyl derivative 14a, never exceeded $73 \%$ in this case. As prolonged treatment in the presence of acid led to the regeneration of diene 1, we assume hydrogen fluoride elimination, followed by a retro-Diels-Alder process and aromatization. The reasonable and quite useful formation of lactone $\mathbf{1 7}$ was observed on treatment with $\mathrm{HCl}$ in tetrahydrofuran, albeit in low yield.

Things became more complicated when we turned to nucleophilic attack to the unsaturated carbonyl group of 9a.

As expected, treatment with just one equivalent of L-Selectride in toluene at $-78{ }^{\circ} \mathrm{C}$ led to the saturated ketone 18 in high yield (Scheme 6). Subsequent reduction with K-Selectride gave rise to the $\alpha$-carbinol 20 exclusively. However, the reduction of 9a with 2,2 equivalents of LSelectride did not even provide a trace of the desired product. To our surprise, the high yield reaction product turned out to be polycyclic ether $\mathbf{1 9}$, which obviously had been formed under extrusion of hydrogen fluoride. 

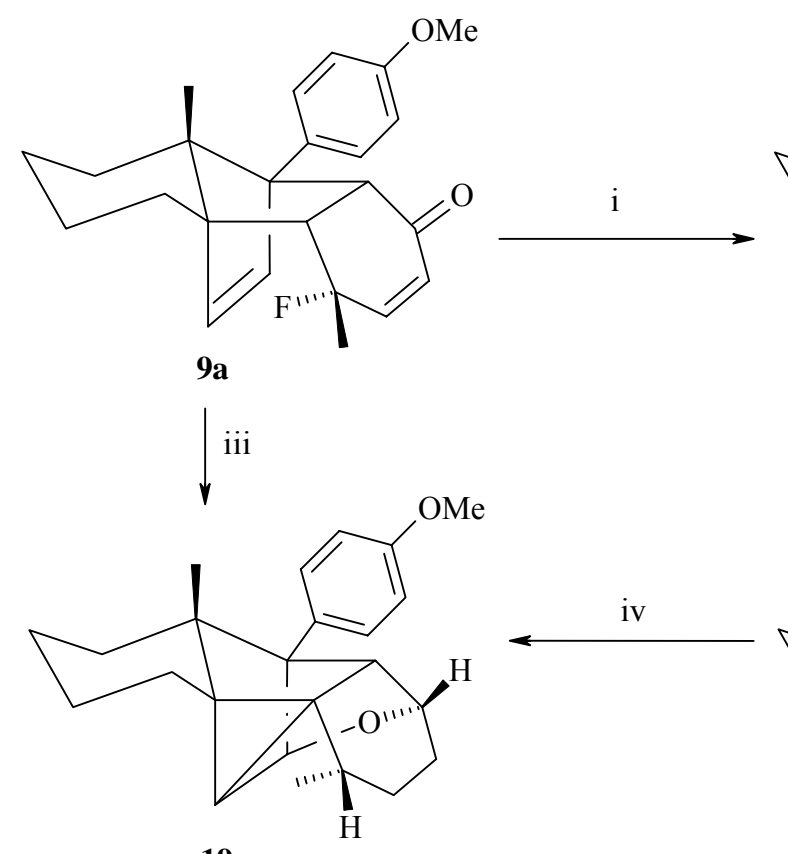

19
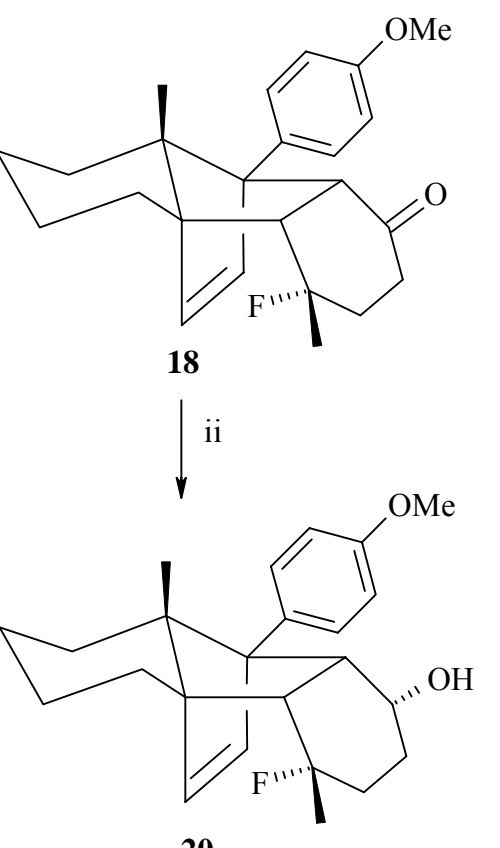

Scheme 6. i: L-Selectride 1 eq., toluene, $-78{ }^{\circ} \mathrm{C}, 15 \mathrm{~min}, 86 \%$; ii: K-Selectride, toluene, $-78{ }^{\circ} \mathrm{C}$, 15 min, $64 \%$; iii: L-Selectride 2.2 eq., toluene, $-78{ }^{\circ} \mathrm{C}, 15$ min, $82 \%$; iv: $\mathrm{LiPF}_{6}, \mathrm{CH}_{2} \mathrm{Cl}_{2}, \mathrm{RT}, 5$ $\min , 49 \%$.

This unusual and on the first glance certainly unexpected structure was confirmed by the facts that no fluoro atom was present anymore, that a hydroxyl group was missing, and that the NMR-signal of the methyl group in the six-membered ring showed up as a doublet, giving rise to the assumption that the fluoro atom had been replaced by hydrogen. Additionally, the double bond in the five-membered ring had disappeared. An X-ray analysis finally proved structure $\mathbf{1 9}$ (Figure 1).

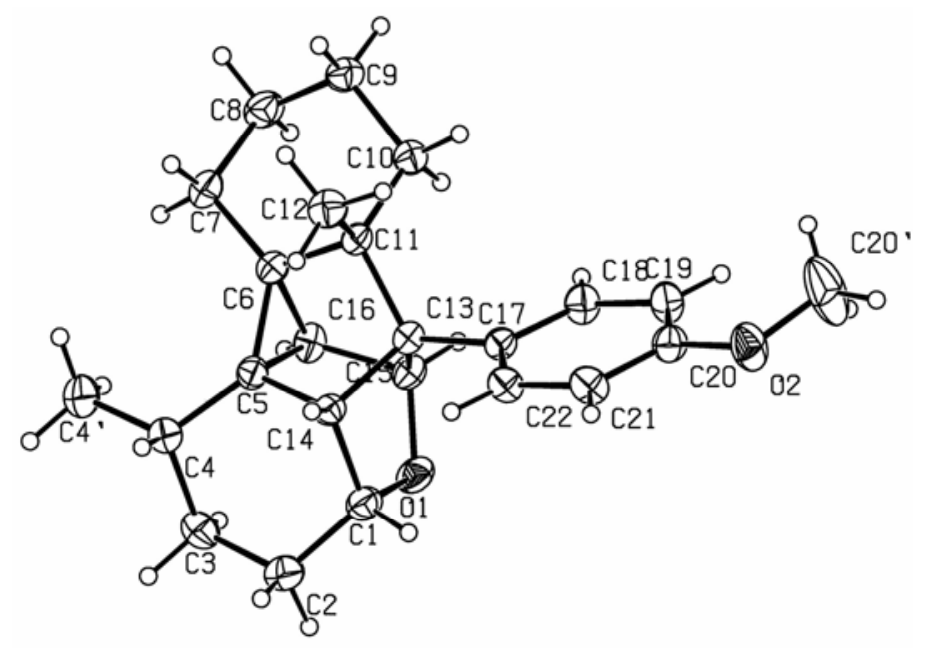

Figure 1. X-ray structure of 19. 
The importance of the lithium counterion for this strange behaviour is clearly demonstrated by the fact that ether formation was also noticed during L-Selectride reduction of the saturated ketone 18, proving this compound to be an intermediate en route to 19. On the other hand, KSelectride reduction of this ketone uneventfully led to the secondary alcohol 20, which on treatment with lithium hexafluorophosphate in dichloromethane was finally converted to ether 19 again.

Obviously, the lithium cation in inert solvents like toluene or dichloromethane operates as a Lewis acid coordinating with the fluorine, which triggers the rearrangement (see 21, Scheme 7). Assuming that formation of ether $\mathbf{1 9}$ is due to the combined action of a Lewis acid and a hydride donor and that the Lewis acid behaviour of the counterion determines the fate of the fluoro atom, one had of course to check on Grignard reagents. Those reagents represent a combination of Lewis acid and nucleophile which on predictable 1,2-addition to the unsaturated ketone 9a should result in a highly reactive tertiary, allylic fluoride (see 22).
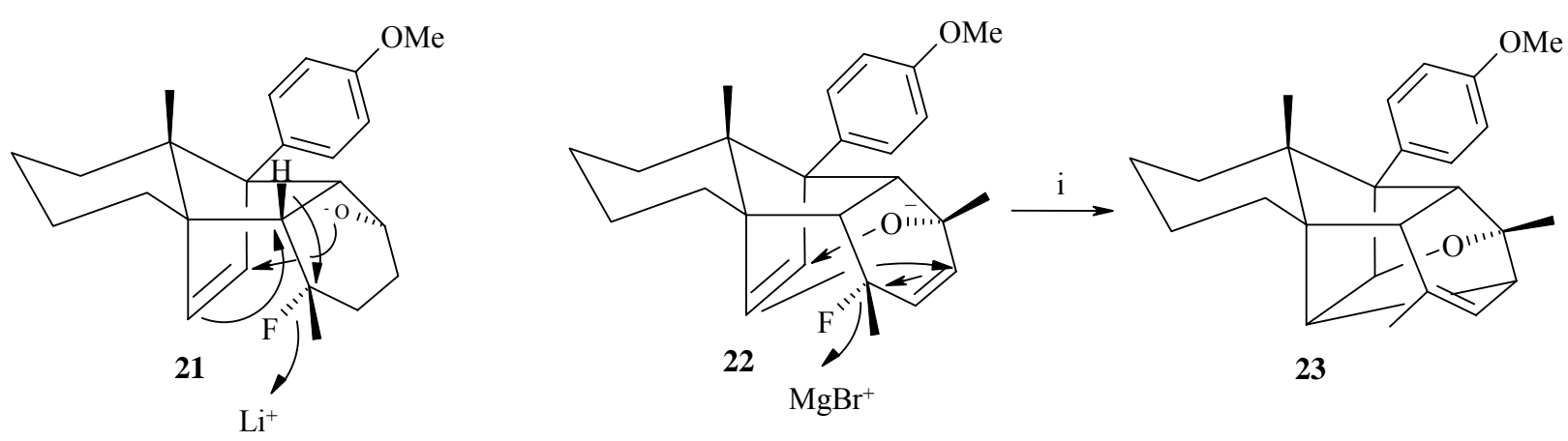

Scheme 7. i: $\mathrm{MeMgBr}$, THF, $-78^{\circ} \mathrm{C}, 5 \mathrm{~min}, 68 \%$.

Accordingly, we were not surprised to again isolate $68 \%$ of a non-fluorinated compound after performing the Grignard reaction at $-78{ }^{\circ} \mathrm{C}$. As $\mathrm{NMR}$ data of this reaction product clearly point to the cyclic ether 23, we assume in this case $\mathrm{SN}^{\prime}$-substitution of the fluoride anion (see 22).

A very similar cyclisation took place in the lithium aluminium hydride reduction of the tertbutyl derivative 9f leading to the cyclic ether 24 (Scheme 8). Although run with a huge excess of lithium aluminium hydride, ether formation in the case of $\mathbf{2 4}$ was a much slower process than the Grignard reaction described above. Additionally, it proved to be a specialty of the sterically hindered tert-butyl compound 9f, which obviously only leads to intramolecular SN'-substitution of the fluoro atom. In contrast 9a under similar conditions gave rise to a mixture of ketone $\mathbf{1 8}$ (66\%) and carbinol 20 (34\%) in quantitative yield via an intermolecular 1,4 - 1,2 hydride addition sequence. These results obtained with a Lewis base reducing agent were compared with the outcome in the case of the Lewis acid reagent DIBAL. Here again $\mathrm{SN}^{\prime}$-substitution dominates the process leading to the homoallylic alcohol 26. It can be assumed that Lewis acid 
assistance from the aluminium alcoholate formed in the reduction step (see 25) is responsible for the outcome of this reaction.
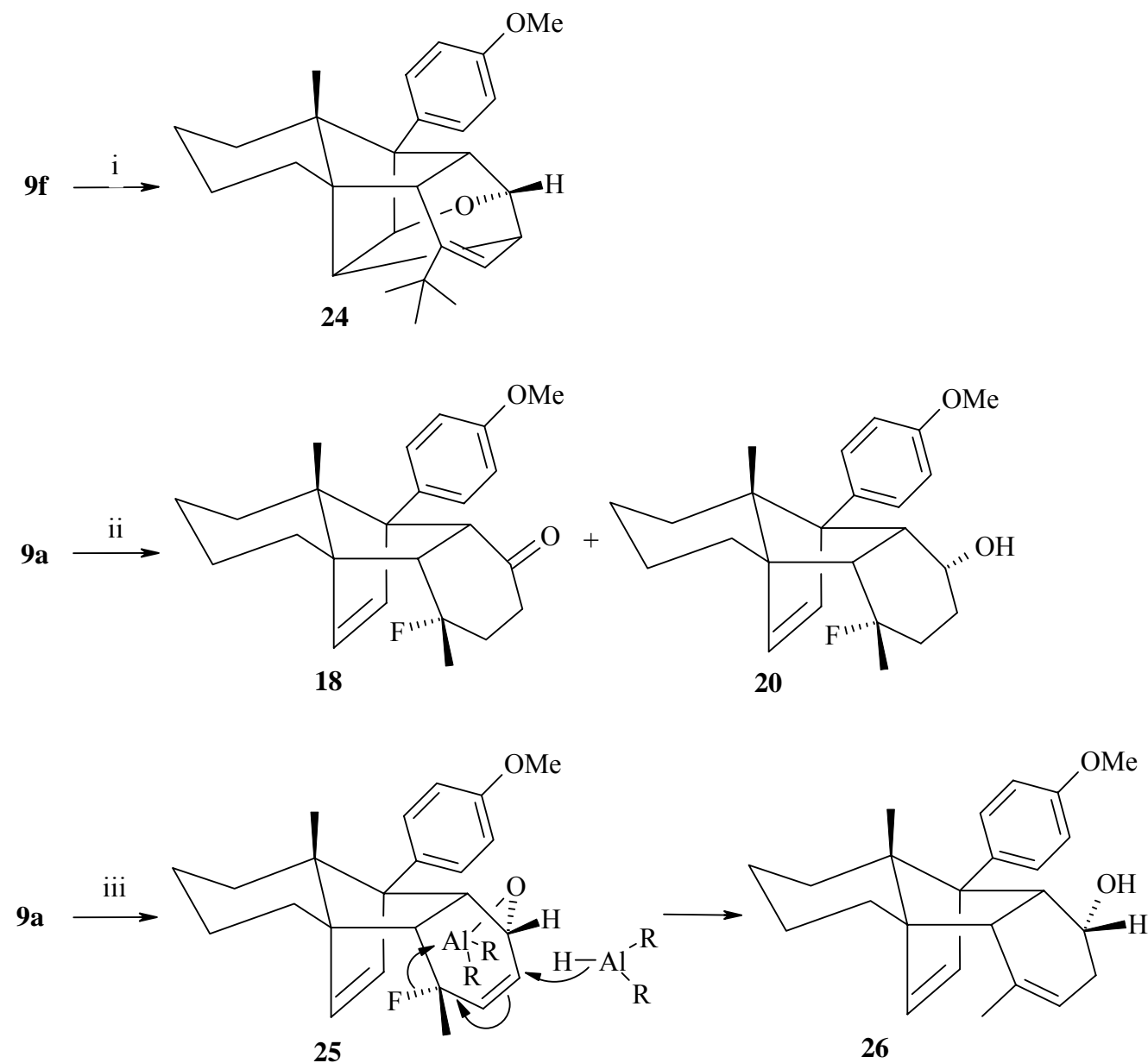

Scheme 8. i: LAH 20 eq., THF, reflux, 1 d, $38 \%$; ii: LAH 1,2 eq., THF, RT, 5 min, 66 \% 18 and 34 \% 20; iii: DIBAL 2.2 eq., toluene, $-78{ }^{\circ} \mathrm{C}, 15 \mathrm{~min}, 76 \%$.

Although we are presenting only a comparatively small selection of results obtained on nucleophilic attack to the carbonyl system it demonstrates the enormous impact of the fluorine on the outcome of this process. Minor changes in the starting material as well as in the reagent may lead to a complete change in the reaction pathway. It would certainly need a profound and detailed investigation of all options to arrive at safe rules and predictions.

\section{Retro Diels-Alder chemistry}

Since all the cyloadducts described so far can be obtained with perfect face selectivity and undergo any subsequent transformation with very high diastereoselectivity, one could expect homochiral fluorine substituted cyclohexenones from their thermal retro Diels-Alder processes. 
While compounds with free hydroxyl groups like $\mathbf{1 3}$ and $\mathbf{1 6}$ failed completely during RetroDiels-Alder processes, good to excellent yields could be obtained starting from protected derivatives 14a, 14b and 15 (Scheme 9).
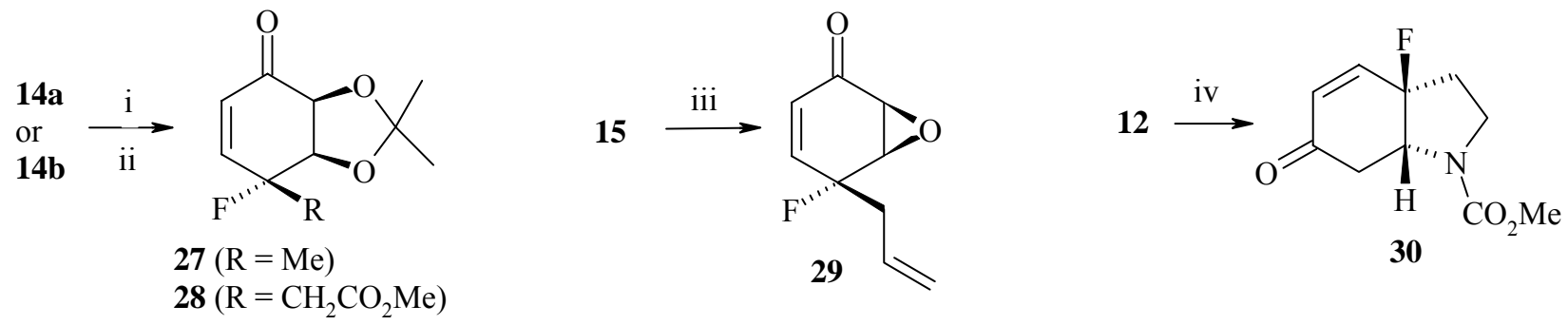

Scheme 9. i: 14a, $2 * 10^{-2} \mathrm{mbar}, 350{ }^{\circ} \mathrm{C}, 76 \%$; ii: 14b, $2 * 10^{-2} \mathrm{mbar}, 350{ }^{\circ} \mathrm{C}, 69 \%$; iii: $2 * 10^{-2}$ mbar, $350{ }^{\circ} \mathrm{C}, 70 \%$; iv: $2 * 10^{-2}$ mbar, $350{ }^{\circ} \mathrm{C}, 100 \%$.

It is worth to mention that the indolinone adduct 12, although suffering from poor yields in its preparation, surprisingly undergoes the retro process in quantitative yield.

\section{Conclusions}

All cycloadditions employing diene 1 and the fluorocyclohexadienones 5a-5f, 7, and 11 have been shown to proceed with excellent regio- and face-selectivity to provide in all cases a single homochiral Diels-Alder adduct.

Subsequent oxidation reactions at the remaining double bond were characterised by exclusive $\beta$-attack leading to reaction products that could be transformed easily into homochiral cyclohexenones in a thermal Retro Diels-Alder reaction.

While these oxidations parallel those with non-fluorinated compounds, diastereoselective nucleophilic attack to the unsaturated carbonyl group led to hydroxyl intermediates, which rearranged under formation of cyclic ethers and under extrusion of the fluoride anion.

\section{Experimental Section}

General Procedures. All solvents were destilled before use. Column chromatography was performed on silica gel (Baker, 30-60 $\mu \mathrm{m}$ ) with the indicated eluent. High pressure of $14 \mathrm{kbar}$ was achieved with a high pressure apparatus from Hofer. Reactions with 6,5 kbar were performed with a high pressure apparatus from Nova Swiss using the pneumatic pump 550.0441A. FVP (flash vacuum pyrolysis) was carried out in an apparatus presented by Seybold und Jersak $^{19}$. Melting points were determined with a melting point apparatus from Gallenkamp and are not corrected. IR-spectra were recorded with a Perkin-Elmer FT 1710 spectrometer. The 
wave numbers $v$ of the characteristic bands are given in $\mathrm{cm}^{-1}$. The intensities are abbreviated as follows: s (strong), $\mathrm{m}$ (middle) und $\mathrm{w}$ (weak). NMR spectra were recorded on a Bruker AM 400 $\left(400 \mathrm{MHz}\right.$ for ${ }^{1} \mathrm{H}, 100 \mathrm{MHz}$ for $\left.{ }^{13} \mathrm{C}\right)$, WP $200\left(200 \mathrm{MHz}\right.$ for $\left.{ }^{1} \mathrm{H}\right)$ and Varian Gemini $200 \mathrm{BB}$ (188 MHz for ${ }^{19} \mathrm{~F}$ ) spectrometer. Chemical shifts $\left(\delta\right.$ in ppm) are given from internal $\mathrm{CHCl}_{3}$ (7.26) for ${ }^{1} \mathrm{H} N M R$ and ${ }^{13} \mathrm{CDCl}_{3}$ (77.0) for ${ }^{13} \mathrm{C}$ NMR. Coupling constants J are presented in Hz. Multiplicities for ${ }^{1} \mathrm{H}$ NMR are abbreviated as follows: s (singlet), d (doublet), tr (triplet), $\mathrm{q}$ (quartet), $\mathrm{m}$ (multiplet). Broad signals are marked with a br. The number of protons is electronically integrated. Multiplicities for ${ }^{13} \mathrm{C}$ NMR have been obtained by using the DEPT (Distortionless Enhancement by Polarisations Transfer) technique. The signals are named as follows: $\mathrm{p}=$ primary, $\mathrm{s}=$ secondary, $\mathrm{t}=$ tertiary und $\mathrm{q}=$ quarternary carbon atoms. Mass spectra (MS) were determined on a Finnigan MAT 312 at an ionisation potential of $70 \mathrm{eV}$ and the indicated temperature. Intensities are in relative relation to the base peak. Mass spectra (HRMS) were performed on a VG AUTOSPEC spectrometer using the peak-matching method. FAB (fast atomic bombardment)-spectra were determined with a VG AUTOSPEC in a m-nitrobenzylic alcohol matrix at low resolution. Intensities are given relative to the base peak.

3-(4-Methoxyphenyl)-4,5,6,7-tetrahydro-3aH-indene (1). For preparation and spectroscopic data, see lit. .

4-Fluoro-4-methylcyclohexa-2,5-dienone (5a). Phenyliodine diacetate (PIDA) (28.6 g, $90.22 \mathrm{mmol}$ ) was dissolved in $\mathrm{CH}_{2} \mathrm{Cl}_{2}(1050 \mathrm{ml})$ and pyridinium poly-hydrogen fluoride (PPHF) $(17.34 \mathrm{ml})$ was added. A solution of p-cresol $(7.5 \mathrm{~g}, 69.4 \mathrm{mmol})$ in $\mathrm{CH}_{2} \mathrm{Cl}_{2}(340 \mathrm{ml})$ was prepared and added dropwise to the PIDA/PPHF-mixture at $0{ }^{\circ} \mathrm{C}$ over a period of 45 minutes. The mixture was stirred for another 10 minutes at $0{ }^{\circ} \mathrm{C}$, then the reaction was stopped by addition of solid potassium carbonate. The stirred suspension was allowed to warm to room temperature, filtered, and the solvent was evaporated under reduced pressure. The crude product was chromatographed on silica gel with petroleum ether/ $\mathrm{Et}_{2} \mathrm{O}(5: 1)$ to give $5 \mathrm{a}(4.292 \mathrm{~g}, 49 \%)$ as a yellow oil. ${ }^{19} \mathrm{~F}-\mathrm{NMR}\left(188 \mathrm{MHz}, \mathrm{CDCl}_{3}\right): \delta=-146.0(\mathrm{~s}, 1 \mathrm{~F})$; For further spectroscopic data, see lit. $^{13}$

4-Allyl-4-fluorocyclohexa-2,5-dienone (5b). PPHF $(0.56 \mathrm{ml})$ was added to a solution of pallylphenol (301 mg, $2.24 \mathrm{mmol})$ in $\mathrm{CH}_{2} \mathrm{Cl}_{2}(80 \mathrm{ml})$ followed by portion wise addition of bistrifluoroacetoxy iodobenzene (PIFA) $(1.235 \mathrm{~g}, 2.912 \mathrm{mmol})$. The reaction was quenched with solid potassium carbonate after 30 minutes of stirring at room temperature. The suspension was stirred for an additional five minutes, filtered, and concentrated in vacuo. Flash chromatography on silica gel with petroleum ether/ $\mathrm{Et}_{2} \mathrm{O}(13: 1)$ afforded $\mathbf{5 b}(123 \mathrm{mg}, 36 \%)$ as a yellow liquid. IR $\left(\mathrm{CHCl}_{3}, \mathrm{~cm}^{-1}\right): 3084(\mathrm{w}), 3028(\mathrm{w}), 2944(\mathrm{w}), 2912$ (w), 1676 (s), 1640 (s), 1384 (m), 1224 (s), 928 (s), 856 (s); ${ }^{1} \mathrm{H}$ NMR (200 MHz, $\left.\mathrm{CDCl}_{3}\right): \delta=2.64(\mathrm{dd}, 16 / 8 \mathrm{~Hz}, 2 \mathrm{H}) ; 5.18(\mathrm{~m}, 2 \mathrm{H}) ; 5.71(\mathrm{~m}$, $1 \mathrm{H}) ; 6.25(\mathrm{~d}, 10 \mathrm{~Hz}, 2 \mathrm{H}) ; 6.88(\mathrm{dd}, 10 / 6 \mathrm{~Hz}, 2 \mathrm{H})$; Exact mass Calcd.: 152.0638, found: 152.0638 .

Methyl(1-fluoro-4-oxocyclohexa-2,5-dien-1-yl)acetate (5c). The reaction was performed according to the preparation of 5a with PIDA (2.481 g, $7.8 \mathrm{mmol})$ in $\mathrm{CH}_{2} \mathrm{Cl}_{2}(80 \mathrm{ml}), \mathrm{PPHF}$ 
(1.5 ml), methyl (4-hydroxyphenyl)acetate $(1 \mathrm{~g}, 6 \mathrm{mmol})$ in $\mathrm{CH}_{2} \mathrm{Cl}_{2}(40 \mathrm{ml})$, and a flash chromatography on silica gel with petroleum ether/Et $\mathrm{E}_{2} \mathrm{O}(5: 1) .5 \mathrm{c}(722 \mathrm{mg}, 65 \%)$ was obtained as a yellow oil. IR $\left(\mathrm{CHCl}_{3}\right): v=3040(\mathrm{w}), 2956(\mathrm{w}), 1720(\mathrm{~s}), 1680(\mathrm{~s}), 1640(\mathrm{~m}), 1436(\mathrm{~m}), 1172$ (m) $\mathrm{cm}^{-1} ;{ }^{1} \mathrm{H}$ NMR $\left(200 \mathrm{MHz}, \mathrm{CDCl}_{3}\right): \delta=2.91(\mathrm{~d}, 17 \mathrm{~Hz}, 2 \mathrm{H}) ; 3.72(\mathrm{~s}, 3 \mathrm{H}) ; 6.29(\mathrm{~d}, 9 \mathrm{~Hz}, 2 \mathrm{H})$, 7.06 (dd, 6/9 Hz, 2H); Exact mass Calcd.: 184.0536, found: 184.0537.

Methyl 1-fluoro-4-oxocyclohexa-2,5-diene-1-carboxylate (5d). The reaction was performed according to the preparation of 5a with bis-trifluoroacetoxy pentafluorophenyliodane (662 $\mathrm{mg}$, $1.3 \mathrm{mmol})$ in $\mathrm{CH}_{2} \mathrm{Cl}_{2}(13 \mathrm{ml})$, PPHF $(0.25 \mathrm{ml})$, methyl 4-hydroxybenzoate $(152 \mathrm{mg}, 1 \mathrm{mmol})$ in $\mathrm{CH}_{2} \mathrm{Cl}_{2}(7 \mathrm{ml})$, and a flash chromatography on silica gel with petroleum ether/Et $2 \mathrm{O}(3: 1)$. 5d (16 mg, $9 \%$ ) was obtained as a white solid. IR $\left(\mathrm{CHCl}_{3}, \mathrm{~cm}^{-1}\right): 3040(\mathrm{w}), 2956(\mathrm{w}), 1764(\mathrm{~s})$, 1676 (s), 1640 (m), 1436 (m), 1256 (s), 1036 (s), 852 (m); ${ }^{1} \mathrm{H}$ NMR (200 MHz, CDCl $): \delta=3.83$ $(\mathrm{s}, 3 \mathrm{H}) ; 6.42(\mathrm{~d}, 10 \mathrm{~Hz}, 2 \mathrm{H}) ; 6.92$ (dd, 10/7 Hz, 2H); Exact mass Calcd.: 170.0379, found: 170.0379 .

Methyl 2-(1-fluoro-4-oxocyclohexa-2,5-diene-1-yl)ethylcarbamate (5e). The reaction was performed according to the preparation of 5a with PIDA (5.666 g, $17.862 \mathrm{mmol})$ in $\mathrm{CH}_{2} \mathrm{Cl}_{2}$ (185 ml), PPHF (3.436 ml), methyl 2-(4-hydroxyphenyl)ethylcarbamate (2.683 g, $13.74 \mathrm{mmol})$ in $\mathrm{CH}_{2} \mathrm{Cl}_{2}(90 \mathrm{ml})$, and a flash chromatography on silica gel with petroleum ether/Et ${ }_{2} \mathrm{O}(5: 1)$. $5 \mathbf{e}$ (773 mg, $22 \%$ ) was obtained as a white solid. IR $\left(\mathrm{CHCl}_{3}, \mathrm{~cm}^{-1}\right): 3456(\mathrm{~m}), 3000(\mathrm{w}), 2956(\mathrm{w})$, 1724 (s), 1676 (s), 1640 (m), 1516 (s), 1236 (s); ${ }^{1} \mathrm{H}$ NMR (400 MHz, $\mathrm{CDCl}_{3}$ ): $\delta=2.14$ (dtr, 17/7 $\mathrm{Hz}, 2 \mathrm{H}) ; 3.32$ (m, 2H); 3.67 (s, 3H); 4.80 (mbr, 1H); 6.27 (d, $10 \mathrm{~Hz}, 2 \mathrm{H}) ; 6.92$ (dd, 10/7 Hz, 2H); Exact mass Calcd.: 213.0801, found: 213.0802 .

4-tert-Butyl-4-fluorocyclohexa-2,5-dienone (5f). The reaction was performed according to the preparation of $5 \mathbf{b}$ with p-tert-butylphenol (5 g, $33.3 \mathrm{mmol}), \mathrm{CH}_{2} \mathrm{Cl}_{2}$ (666 ml), PPHF (8.32 ml), PIDA (13.937 $\mathrm{g}, 43.29 \mathrm{mmol})$, and a flash chromatography on silica gel with petroleum ether/ $\mathrm{Et}_{2} \mathrm{O}(10: 1)$. $5 \mathbf{f}(381 \mathrm{mg}, 7 \%)$ was obtained as a yellow liquid. IR $\left(\mathrm{CHCl}_{3}, \mathrm{~cm}^{-1}\right): 2972(\mathrm{~m})$, 1676 (s), 1636 (s), 1504 (w), 1368 (m), 1172 (m), 992 (m); ${ }^{1} \mathrm{H}$ NMR (400 MHz, $\mathrm{CDCl}_{3}$ ): $\delta=$ 1.06 (d, $1 \mathrm{~Hz}, 9 \mathrm{H}) ; 6.29$ (d, $10 \mathrm{~Hz}, 2 \mathrm{H}) ; 7.00$ (dd, 7/10 Hz, 2H); Exact mass Calcd.: 168.0953, found: 168.0950 .

7a-Fluoro-1,2,3,7a-tetrahydro-5H-inden-5-one (7). The reaction was performed according to the preparation of 5a with PIDA (3.073 g, $9.689 \mathrm{mmol})$ in $\mathrm{CH}_{2} \mathrm{Cl}_{2}(100 \mathrm{ml})$, PPHF (1.86 ml), 5indanol $(1 \mathrm{~g}, 7.453 \mathrm{mmol})$ in $\mathrm{CH}_{2} \mathrm{Cl}_{2}(50 \mathrm{ml})$, and a flash chromatography on silica gel with petroleum ether/ $\mathrm{Et}_{2} \mathrm{O}(10: 1) .7$ (537 mg, $47 \%$ ) was obtained as a yellow oil. For spectroscopic data, see Lit. $^{13}$

4-Hydroxy-4-methylcyclohexa-2,5-dien-1-one (8). For preparation and spectroscopic data, see Lit. $^{7}$

4-Fluoro-4-methylcyclohexa-2,5-dienone adduct (9a). Dienone 5a (50 mg, $0.396 \mathrm{mmol}$ ), diene 1 (105 mg, $0.436 \mathrm{mmol})$, TEMPO (93 mg, $0.436 \mathrm{mmol})$ and $\mathrm{CH}_{2} \mathrm{Cl}_{2}(0.5 \mathrm{ml})$ were sealed in a Teflon tube and exposed to a pressure of $14 \mathrm{kbar}$ for 14 days. After evaporating the solvent under reduced pressure and flash chromatography on silica gel with petroleum ether/ $\mathrm{Et}_{2} \mathrm{O}(5: 1)$ 9a 
(130 mg, $90 \%$ ) was obtained as a white solid. ${ }^{19} \mathrm{~F}-\mathrm{NMR}\left(188 \mathrm{MHz}, \mathrm{CDCl}_{3}\right): \delta=-141.7(\mathrm{~s}, 1 \mathrm{~F})$; For further spectroscopic data, see Lit. ${ }^{5}$

4-Allyl-4-fluorocyclohexa-2,5-dienone adduct (9b). The reaction was performed according to the preparation of 9a. with dienone $5 \mathbf{b}(200 \mathrm{mg}, 1.314 \mathrm{mmol})$, diene 1 (348 $\mathrm{mg}, 1.445 \mathrm{mmol})$, TEMPO (308 mg, $1.445 \mathrm{mmol}), \mathrm{CH}_{2} \mathrm{Cl}_{2}(1.3 \mathrm{ml})$ over a period of $7 \mathrm{~d}$ at $14 \mathrm{kbar}$ followed by a flash chromatography on silica gel with petroleum ether/ $\mathrm{Et}_{2} \mathrm{O}(5: 1)$. 9b (513 $\left.\mathrm{mg}, 100 \%\right)$ was obtained as a white foam. IR $\left(\mathrm{CHCl}_{3}, \mathrm{~cm}^{-1}\right): 3000(\mathrm{w}), 2928(\mathrm{~m}), 2864(\mathrm{w}), 1668(\mathrm{~s}), 1640(\mathrm{w})$, 1612 (w), 1516 (s), 1252 (s), 1180 (s); ${ }^{1} \mathrm{H}$ NMR (200 MHz, $\mathrm{CDCl}_{3}$ ): $\delta=0.45$ (dbr, $\left.13 \mathrm{~Hz}, 1 \mathrm{H}\right)$; $0.81(\mathrm{~s}, 3 \mathrm{H}) ; 1.03-1.71(\mathrm{~m}, 5 \mathrm{H}) ; 1.84$ (dtrbr, 13/4 Hz, 1H); 2.28 (dbr, $10 \mathrm{~Hz}, 1 \mathrm{H}) ; 2.51$ (m, 2H); $2.88(\mathrm{~d}, 9 \mathrm{~Hz}, 1 \mathrm{H}) ; 3.67(\mathrm{dd}, 9 / 5 \mathrm{~Hz}, 1 \mathrm{H}) ; 3.80(\mathrm{~s}, 3 \mathrm{H}) ; 5.14(\mathrm{~m}, 1 \mathrm{H}) ; 5.23(\mathrm{~m}, 1 \mathrm{H}) ; 5.66(\mathrm{~m}$, $1 \mathrm{H}) ; 5.82(\mathrm{~d}, 12 \mathrm{~Hz}, 1 \mathrm{H}) ; 5.84(\mathrm{~d}, 6 \mathrm{~Hz}, 1 \mathrm{H}) ; 6.06(\mathrm{dd}, 6 / 4 \mathrm{~Hz}, 1 \mathrm{H}) ; 6.47(\mathrm{dd}, 12 / 11 \mathrm{~Hz}, 1 \mathrm{H})$; $6.88(\mathrm{~d}, 9 \mathrm{~Hz}, 2 \mathrm{H}) ; 7.31$ (d, $9 \mathrm{~Hz}, 2 \mathrm{H})$; FAB-MS: m/z (\%)=392 (M+1 3), 240 (100, diene 1).

Methyl(1-fluoro-4-oxocyclohexa-2,5-dien-1-yl)acetate adduct (9c). The reaction was performed according to the preparation of 9a with dienone 5c (1.872 g, $10.165 \mathrm{mmol})$, diene 1 (2.687 g, $11.182 \mathrm{mmol})$, TEMPO (2.385 g, $11.182 \mathrm{mmol}), \mathrm{CH}_{2} \mathrm{Cl}_{2}(10 \mathrm{ml})$ over a period of $10 \mathrm{~d}$ at $6.5 \mathrm{kbar}$ followed by a flash chromatography on silica gel with petroleum ether/ $\mathrm{Et}_{2} \mathrm{O}(3: 1)$. 9c (3.263 g, $76 \%$ ) was obtained as a white solid, mp 91.6 ${ }^{\circ} \mathrm{C}$. IR $\left(\mathrm{CHCl}_{3}, \mathrm{~cm}^{-1}\right): 3000(\mathrm{w}), 2952$ (m), 2928 (m), 2864 (w), 1740 (s), 1672 (s), 1612 (w), 1516 (s), 1248 (s), 1180 (s); ${ }^{1} \mathrm{H}$ NMR $\left(200 \mathrm{MHz}, \mathrm{CDCl}_{3}\right): \delta=0.46(\mathrm{dbr}, 13 \mathrm{~Hz}, 1 \mathrm{H}) ; 0.83(\mathrm{~s}, 3 \mathrm{H}) ; 1.12-1.72(\mathrm{~m}, 5 \mathrm{H}) ; 1.85$ (dtrbr, 14/4 $\mathrm{Hz}, 1 \mathrm{H}) ; 2.27$ (dbr, $13 \mathrm{~Hz}, 1 \mathrm{H}) ; 2.86$ (dd, 14/2 Hz, 2H); 3.11 (dd, 8/3 Hz, 1H); 3.67 (s, 3H); 3.78 $(\mathrm{d}, 8 \mathrm{~Hz}, 1 \mathrm{H}) ; 3.80(\mathrm{~s}, 3 \mathrm{H}) ; 5.85(\mathrm{dd}, 10 / 2 \mathrm{~Hz}, 1 \mathrm{H}) ; 5.86(\mathrm{~d}, 5 \mathrm{~Hz}, 1 \mathrm{H}) ; 6.05(\mathrm{dd}, 5 / 6 \mathrm{~Hz}, 1 \mathrm{H})$; 6.51 (dd, 13/10 Hz, 1H); 6.87 (d, $9 \mathrm{~Hz}, 2 \mathrm{H}) ; 7.32$ (dd, 9/9 Hz, 2H); NOE-experiment:

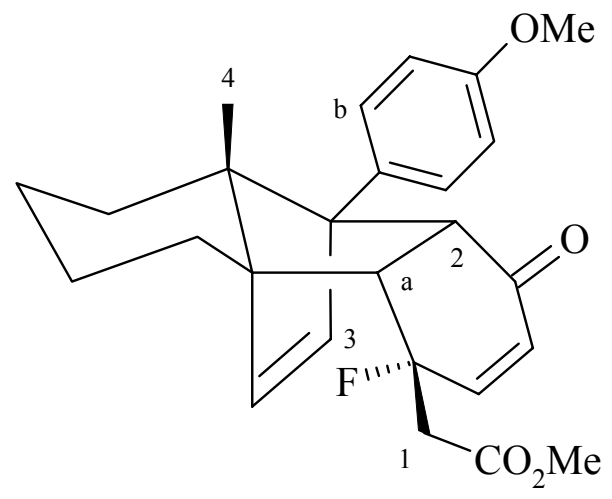

Irradiation: $\quad$ NOE:

$\mathrm{H}-\mathrm{a} \quad+6,0 \%(\mathrm{H}-1),+10,2 \%(\mathrm{H}-4)$

$\mathrm{H}-\mathrm{b} \quad+9,0 \%(\mathrm{H}-2),+8,2 \%(\mathrm{H}-3),+2,5 \%(\mathrm{H}-4)$.

Exact mass Calcd.: 424.2049, found: 424,2052.

Methyl 1-fluoro-4-oxocyclohexa-2,5-diene-1-carboxylate adduct (9d). The reaction was performed according to the preparation of 9a with dienone $5 \mathbf{d}$ (52 $\mathrm{mg}, 0.297 \mathrm{mmol})$, diene $\mathbf{1}$ 
(81 mg, $0.327 \mathrm{mmol}), \mathrm{CH}_{2} \mathrm{Cl}_{2}(0.5 \mathrm{ml})$ over a period of $7 \mathrm{~d}$ at $6.5 \mathrm{kbar}$ followed by a flash chromatography on silica gel with petroleum ether/ $\mathrm{Et}_{2} \mathrm{O}(3: 1)$. 9d (101 mg, $\left.81 \%\right)$ was obtained as a white solid, mp $139.9^{\circ} \mathrm{C}$. IR $\left(\mathrm{CHCl}_{3}, \mathrm{~cm}^{-1}\right): 3000(\mathrm{w}), 2956(\mathrm{~m}), 2936(\mathrm{~m}), 2864(\mathrm{w}), 1756$ (s), 1672 (s), 1636 (w), 1516 (s), 1252 (s), 1032 (m); ${ }^{1} \mathrm{H}$ NMR (400 MHz, $\mathrm{CDCl}_{3}$ ): $\delta=0.48$ (dbr, $13 \mathrm{~Hz}, 1 \mathrm{H}) ; 0.83$ (s, 3H); 1.12-1.49 (m, 4H); 1.64 (dbr, $13 \mathrm{~Hz}, 1 \mathrm{H}) ; 1.85$ (dtrbr, 13/4 Hz, 1H); 2.28 (ddbr, 13/2 Hz, 1H); 3.15 (d, $8 \mathrm{~Hz}, 1 \mathrm{H}) ; 3.80$ (s, 6H); 3.90 (dd, 8/4 Hz, 1H); 5.89 (d, $6 \mathrm{~Hz}$, $1 \mathrm{H}) ; 5.95(\mathrm{~d}, 10 \mathrm{~Hz}, 1 \mathrm{H}) ; 6.07(\mathrm{dd}, 6 / 3 \mathrm{~Hz}, 1 \mathrm{H}) ; 6.42(\mathrm{dd}, 13 / 10 \mathrm{~Hz}, 1 \mathrm{H}) ; 6.88(\mathrm{~d}, 9 \mathrm{~Hz}, 2 \mathrm{H})$; 7.32 (d, $9 \mathrm{~Hz}, 2 \mathrm{H}) ;{ }^{13} \mathrm{C}$ NMR $\left(100 \mathrm{MHz}, \mathrm{CDCl}_{3}\right): \delta=15.26(\mathrm{p}) ; 21.03(\mathrm{~s}) ; 23.72$ (s); 27.40 (s); 28.42 (s); 47.17 (t, doublet $20 \mathrm{~Hz}) ; 49.94$ (t); 53.58 (p); 55.18 (p); 61.64 (q); 62.39 (q); 71.17 (q); 93.02 (q, doublet $190 \mathrm{~Hz}) ; 2 \mathrm{x} 113.13$ (t);2x $128.60(\mathrm{q}) ; 129.13(\mathrm{t}), 134.23(\mathrm{t}) ; 136.27(\mathrm{t}) ; 138.60$ $(\mathrm{t}) ; 140.85(\mathrm{t}$, doublet $31 \mathrm{~Hz}) ; 158.41(\mathrm{q}) ; 170.48$ (q, doublet $29 \mathrm{~Hz}) ; 197.69$ (q); Exact mass Calcd.: 410.1893, found: 410.1878 .

Methyl 2-(1-fluoro-4-oxocyclohexa-2,5-diene-1-yl)ethylcarbamate adduct (9e). The reaction was performed according to the preparation of 9a with dienone $5 \mathbf{e}(141 \mathrm{mg}, 0.661 \mathrm{mmol})$, diene $1(175 \mathrm{mg}, 0.727 \mathrm{mmol}), \mathrm{CH}_{2} \mathrm{Cl}_{2}(0.7 \mathrm{ml})$ over a period of $14 \mathrm{~d}$ at $6.5 \mathrm{kbar}$ followed by a flash chromatography on silica gel with petroleum ether/ $\mathrm{Et}_{2} \mathrm{O}(1: 3)$. 9e (226 mg, $75 \%$ ) was obtained as a colorless oil. IR $\left(\mathrm{CHCl}_{3}, \mathrm{~cm}^{-1}\right): 3456(\mathrm{~m}), 3000(\mathrm{w}), 2956(\mathrm{~m}), 2928(\mathrm{~m}), 2860(\mathrm{w}), 1720$ (s), 1672 (s), 1526 (s), 1240 (s); ${ }^{1} \mathrm{H}$ NMR (400 MHz, $\mathrm{CDCl}_{3}$ ): $\delta=0.45$ (dbr, $13 \mathrm{~Hz}, 1 \mathrm{H}$ ); 0.83 (s, $3 \mathrm{H}) ; 1.12-2.32(\mathrm{~m}, 9 \mathrm{H}) ; 2.89$ (d, $8 \mathrm{~Hz}, 1 \mathrm{H}) ; 3.33$ (m, 2H); 3.67 (s, 3H); 3.75 (dd, 8/5 Hz, 1H); $4.84(\mathrm{~m}, 1 \mathrm{H}) ; 5.81(\mathrm{~d}, 10 \mathrm{~Hz}, 1 \mathrm{H}) ; 5.84(\mathrm{~d}, 6 \mathrm{~Hz}, 1 \mathrm{H}) ; 6.52(\mathrm{dd}, 10 / 11 \mathrm{~Hz}, 1 \mathrm{H}) ; 6.88(\mathrm{~d}, 9 \mathrm{~Hz}$, 2H); 7.30 (d, 9 Hz, 2H); FAB-MS: m/z (\%)= $453\left(\mathrm{M}^{+}, 2\right), 240$ (100, diene 1).

4-tert-Butyl-4-fluorocyclohexa-2,5-dienone adduct (9f). The reaction was performed according to the preparation of 9a with dienone $5 f(50 \mathrm{mg}, 0.297 \mathrm{mmol})$, diene 1 (79 $\mathrm{mg}, 0.327 \mathrm{mmol})$, $\mathrm{CH}_{2} \mathrm{Cl}_{2}(0.5 \mathrm{ml})$ ) over a period of $14 \mathrm{~d}$ at $6.5 \mathrm{kbar}$ followed by a flash chromatography on silica gel with petroleum ether/ $\mathrm{Et}_{2} \mathrm{O}(5: 1)$. 9f $(87 \mathrm{mg}, 72 \%)$ was obtained as a white foam. IR $\left(\mathrm{CHCl}_{3}\right.$, $\mathrm{cm}^{-1}$ ): 2960 (s), 2928 (s), 2864 (m), 1668 (s), 1612 (w), 1516 (s), 1248 (m), 1180 (m); ${ }^{1} \mathrm{H}$ NMR $\left(200 \mathrm{MHz}, \mathrm{CDCl}_{3}\right): \delta=0.44(\mathrm{dbr}, 13 \mathrm{~Hz}, 1 \mathrm{H}) ; 0.85(\mathrm{~d}, 1 \mathrm{~Hz}, 3 \mathrm{H}) ; 0.96(\mathrm{~d}, 1 \mathrm{~Hz}, 9 \mathrm{H}) ; 1.08-1.73$ $(\mathrm{m}, 5 \mathrm{H}) ; 1.85(\mathrm{~m}, 1 \mathrm{H}) ; 2.26(\mathrm{~m}, 1 \mathrm{H}) ; 3.01(\mathrm{~d}, 8 \mathrm{~Hz}, 1 \mathrm{H}) ; 3.63(\mathrm{dd}, 8 / 6 \mathrm{~Hz}, 1 \mathrm{H}) ; 3.80(\mathrm{~s}, 3 \mathrm{H})$; 5.81 (d, $6 \mathrm{~Hz}, 1 \mathrm{H}) ; 5.91$ (dd, 10/2 Hz, 1H); 6.03 (dd, 6/4 Hz, 1H); 6.46 (dd, 14/10 Hz, 1H); 6.88 (d, $9 \mathrm{~Hz}, 2 \mathrm{H}) ; 7.33$ (dd, 9/10 Hz, 2H); FAB-MS: m/z (\%) = $408\left(\mathrm{M}^{+}, 1\right), 240$ (100, diene 1).

7a-Fluoro-1,2,3,7a-tetrahydro-5 $H$-inden-5-one adduct (10). The reaction was performed according to the preparation of 9a with dienone 7 (241 $\mathrm{mg}, 1.584 \mathrm{mmol})$, diene 1 (419 $\mathrm{mg}$, $1.742 \mathrm{mmol}), \mathrm{CH}_{2} \mathrm{Cl}_{2}(2 \mathrm{ml})$ over a period of $9 \mathrm{~d}$ at $6.5 \mathrm{kbar}$ followed by a flash chromatography on silica gel with petroleum ether/Et $\mathrm{E}_{2} \mathrm{O}(5: 1) .10(86 \mathrm{mg}, 14 \%)$ was obtained as a yellow foam. IR $\left(\mathrm{CHCl}_{3}, \mathrm{~cm}^{-1}\right): 3000$ (m), 2956 (s), 2932 (s), 2868 (m), 1652 (s), 1612 (m), 1516 (s), 1252 (s); ${ }^{1} \mathrm{H}$ NMR $\left(400 \mathrm{MHz}, \mathrm{CDCl}_{3}\right): \delta=0.47(\mathrm{dbr}, 13 \mathrm{~Hz}, 1 \mathrm{H}) ; 0.86(\mathrm{~s}, 3 \mathrm{H}) ; 1.08-2.95(\mathrm{~m}, 13 \mathrm{H}) ; 3.06$ $(\mathrm{dd}, 31 / 8 \mathrm{~Hz}, 1 \mathrm{H}) ; 3.80$ (s, 3H); $3.86(\mathrm{~d}, 8 \mathrm{~Hz}, 1 \mathrm{H}) ; 5.81$ (d, $6 \mathrm{~Hz}, 1 \mathrm{H}) ; 5.90(\mathrm{~m}, 2 \mathrm{H}) ; 6.88$ (d, 10 $\mathrm{Hz}, 2 \mathrm{H}) ; 7.33$ (d, $10 \mathrm{~Hz}, 2 \mathrm{H})$; Exact mass Calcd.: $372.2089\left(\mathrm{M}^{+}-\mathrm{HF}\right)$, found: $372.2084\left(\mathrm{M}^{+}-\mathrm{HF}\right)$.

Methyl 3a-fluoro-6-oxo-2,3,3a,6,7,7a-hexahydro-1H-indole-1-carboxylate (11). Carbamate 5e $(1.85 \mathrm{~g}, 8.677 \mathrm{mmol})$ was dissolved in DMSO $(260 \mathrm{ml})$ and saturated aqueous sodium 
bicarbonate solution $(37 \mathrm{ml})$ was added. The suspension was stirred overnight at $40{ }^{\circ} \mathrm{C}$. After cooling to room temperature, the mixture was partitioned between water and $\mathrm{Et}_{2} \mathrm{O}$. The aqueous layer was separated, neutralized with $2 \mathrm{~N} \mathrm{HCl}$, and extracted four times with $\mathrm{Et}_{2} \mathrm{O}$. The combined organic extracts were washed with brine, dried over $\mathrm{MgSO}_{4}$, filtered, and concentrated in vacuo. The following flash chromatography on silica gel with petroleum ether/Et ${ }_{2} \mathrm{O}(1: 1)$ gave $11(1,215 \mathrm{~g}, 66 \%)$ as a white solid, $\mathrm{mp} 65.2^{\circ} \mathrm{C}$. IR $\left(\mathrm{CHCl}_{3}, \mathrm{~cm}^{-1}\right): 3028(\mathrm{~m}), 2956(\mathrm{~m}), 2900$ (w), 1692 (s), 1452 (s), 1392 (s), 1236 (s), 1124 (s), 948 (s); ${ }^{1} \mathrm{H}$ NMR (400 MHz, CDCl 3 ): $\delta=$ $2.23(\mathrm{~m}, 2 \mathrm{H}) ; 2.44(\mathrm{~m}, 1 \mathrm{H}) ; 3.15(\mathrm{dd}, 16 / 7 \mathrm{~Hz}, 1 \mathrm{H}) ; 3.70(\mathrm{~m}, 2 \mathrm{H}) ; 3.74(\mathrm{~s}, 3 \mathrm{H}) ; 4.42(\mathrm{~m}, 1 \mathrm{H})$; $6.11(\mathrm{~d}, 11 \mathrm{~Hz}, 1 \mathrm{H}) ; 6.89$ (dd, 11/10 Hz, 1H); Exact mass Calcd.: 213.0801, found: 213.0804.

Methyl 3a-fluoro-6-oxo-2,3,3a,6,7,7a-hexahydro-1H-indole-1-carboxylate adduct (12). The reaction was performed according to the preparation of 9a with hydroindole 11 (100 $\mathrm{mg}$, $0.47 \mathrm{mmol})$, diene $1(124 \mathrm{mg}, 0.517 \mathrm{mmol}), \mathrm{CH}_{2} \mathrm{Cl}_{2}(0.5 \mathrm{ml})$ over a period of $14 \mathrm{~d}$ at $14 \mathrm{kbar}$ followed by a flash chromatography on silica gel with petroleum ether/ $\mathrm{Et}_{2} \mathrm{O}$ (1:3). 12 (76 mg, $36 \%$ ) was obtained as a white solid. IR $\left(\mathrm{CHCl}_{3}, \mathrm{~cm}^{-1}\right): 3000(\mathrm{w}), 2956(\mathrm{~m}), 2928(\mathrm{~m}), 2864(\mathrm{w})$, 1692 (s), 1612 (w), 1516 (m), 1456 (s), 1392 (s), 1248 (m), 1228 (m); ${ }^{1} \mathrm{H}$ NMR (400 MHz, $\left.\mathrm{CDCl}_{3}\right): \delta=0.45(\mathrm{dbr}, 13 \mathrm{~Hz}, 1 \mathrm{H}) ; 0.79(\mathrm{~s}, 3 \mathrm{H}) ; 1.14-2.37(\mathrm{~m}, 10 \mathrm{H}) ; 2.79(\mathrm{dd}, 17 / 8 \mathrm{~Hz}, 1 \mathrm{H})$; $2.95(\mathrm{~m}, 1 \mathrm{H}) ; 3.46(\mathrm{~m}, 2 \mathrm{H}) ; 3.68(\mathrm{~s}, 3 \mathrm{H}) ; 3.75(\mathrm{~d}, 10 \mathrm{~Hz}, 1 \mathrm{H}) ; 3.79(\mathrm{~s}, 3 \mathrm{H}) ; 4.02(\mathrm{~m}, 1 \mathrm{H}) ; 6.21$ $(\mathrm{d}, 6 \mathrm{~Hz}, 1 \mathrm{H}) ; 6.31(\mathrm{dd}, 6 / 3 \mathrm{~Hz}, 1 \mathrm{H}) ; 6.87(\mathrm{~d}, 10 \mathrm{~Hz}, 2 \mathrm{H}) ; 7.23(\mathrm{dd}, 10 / 11 \mathrm{~Hz}, 2 \mathrm{H}) ;{ }^{13} \mathrm{C}$ NMR $\left(100 \mathrm{MHz}, \mathrm{CDCl}_{3}\right): \delta=15.31(\mathrm{p}) ; 21.13(\mathrm{~s}) ; 23.74(\mathrm{~s}) ; 26.91(\mathrm{~s}) ; 27.38(\mathrm{~s}) ; 37.25$ (s, doublet 25 $\mathrm{Hz}) ; 41.98(\mathrm{~s}) ; 46.42(\mathrm{~s}) ; 49.82(\mathrm{t}) ; 52.45(\mathrm{p}) ; 54.58(\mathrm{t}) ; 55.16(\mathrm{p}) ; 59.94(\mathrm{t}) ; 62.09$ (q); $62.87(\mathrm{q})$; 68.59 (q); 101.63 (q, doublet 76 Hz); 2x 113.28 (t);2x 128.49 (t); 129.10 (q); 136.90 (t); 139.37 (t); 155.00 (q); 158.34 (q); 208.93 (q); Exact mass Calcd.: 453.2315, found: 453.2325.

Diol adduct (13). For preparation and spectroscopic data, see Lit. ${ }^{13}$.

Acetonide adduct (14a). To a solution of diol adduct $13(102 \mathrm{mg}, 0.255 \mathrm{mmol})$ and a catalytic amount of p-toluenesulfonic acid in dry DMF $(5 \mathrm{ml})$ 2-methoxypropene (120 $\mu 1,1.275 \mathrm{mmol})$ was added at $0{ }^{\circ} \mathrm{C}$. The reaction was stirred over night at room temperature and then diluted with $\mathrm{Et}_{2} \mathrm{O}$. The organic layer was washed two times with brine, dried over $\mathrm{MgSO}_{4}$, and filtered. Concentration in vacuo and subsequent flash chromatography on silica gel with petroleum ether/Et $\mathrm{E}_{2} \mathrm{O}(3: 1)$ provided 14a $(111 \mathrm{mg}, 99 \%)$ as a white solid, $\mathrm{mp} 163.9^{\circ} \mathrm{C}$. IR $\left(\mathrm{CHCl}_{3}, \mathrm{~cm}^{-1}\right)$ : 2996 (s), 2936 (s), 1728 (s), 1612 (w), 1516 (s), 1464 (m), 1380 (s), 1292 (s), 1180 (s), 1072 (s); ${ }^{1} \mathrm{H}$ NMR $\left(400 \mathrm{MHz}, \mathrm{CDCl}_{3}\right): \delta=0.60(\mathrm{dbr}, 13 \mathrm{~Hz}, 1 \mathrm{H}) ; 0.79(\mathrm{~s}, 3 \mathrm{H}) ; 1.10-1.70(\mathrm{~m}, 5 \mathrm{H}) ; 1.38(\mathrm{~s}$, $3 \mathrm{H}) ; 1.57$ (s, 3H); 1.70 (s, 3H); 2.05 (dtrbr, 10/4 Hz, 1H); 2.20 (dbr, $13 \mathrm{~Hz}, 1 \mathrm{H}) ; 3.00$ (dd, 34/10 $\mathrm{Hz}, 1 \mathrm{H}) ; 3.79$ (s, 3H); 4.14 (d, $8 \mathrm{~Hz}, 1 \mathrm{H}) ; 4.28$ (d, $10 \mathrm{~Hz}, 1 \mathrm{H}) ; 4.30$ (dd, 8/5 Hz, 1H); 5.95 (dd, 9/6 Hz, 1H); $6.16(\mathrm{~d}, 6 \mathrm{~Hz}, 1 \mathrm{H}) ; 6.85$ (d, $10 \mathrm{~Hz}, 2 \mathrm{H}) ; 7.15$ (d, $10 \mathrm{~Hz}, 2 \mathrm{H})$; Exact mass Calcd.: 440.2363, found: 440.2368.

Acetonide adduct (14b). Diol 16 (200 $\mathrm{mg}, 0.44 \mathrm{mmol})$ in dry DMF (8 ml), 2,2dimethoxypropane $(4 \mathrm{ml})$ and a catalytic amount of p-toluenesulfonic acid were heated for 1 hour at $100{ }^{\circ} \mathrm{C}$. The work-up procedure started by diluting with $\mathrm{Et}_{2} \mathrm{O}$, washing with water followed by washing with brine. After drying the organic layer over $\mathrm{MgSO}_{4}$, filtering and evaporating the solvent under reduced pressure the crude product was purified by flash 
chromatography on silica gel with petroleum ether/Et ${ }_{2} \mathrm{O}(3: 1)$ to afford $\mathbf{1 4 b}(158 \mathrm{mg}, 73 \%)$ as a white foam. IR $\left(\mathrm{CHCl}_{3}, \mathrm{~cm}^{-1}\right): 2992$ (m), 2932 (s), 2856 (m), 1732 (s), 1612 (w), 1516 (s), 1436 (w), 1384 (m), 1252 (s), 1180 (s); ${ }^{1} \mathrm{H}$ NMR (200 MHz, $\left.\mathrm{CDCl}_{3}\right): \delta=0.60$ (dbr, $\left.13 \mathrm{~Hz}, 1 \mathrm{H}\right) ; 0.78$ $(\mathrm{s}, 3 \mathrm{H}) ; 1.0-2.27(\mathrm{~m}, 7 \mathrm{H}) ; 1.40(\mathrm{~d}, 10 \mathrm{~Hz}, 3 \mathrm{H}) ; 1.60(\mathrm{~d}, 12 \mathrm{~Hz}, 3 \mathrm{H}) ; 2.80(\mathrm{~m}, 3 \mathrm{H}) ; 3.72(\mathrm{~s}, 3 \mathrm{H})$; 3.79 (s, 3H); 4.14 (d, $8 \mathrm{~Hz}, 1 \mathrm{H}) ; 4.32$ (d, $10 \mathrm{~Hz}, 1 \mathrm{H}) ; 4.82$ (dd, 8/6 Hz, 1H); 5.95 (dd, 9/6 Hz, $1 \mathrm{H}) ; 6.20(\mathrm{~d}, 6 \mathrm{~Hz}, 1 \mathrm{H}) ; 6.85$ (d, $9 \mathrm{~Hz}, 2 \mathrm{H}) ; 7.14$ (d, $9 \mathrm{~Hz}, 2 \mathrm{H})$; FAB-MS: m/z (\%) = $498\left(\mathrm{M}^{+}, 6\right)$, 240 (100, diene 1).

Epoxide adduct (15). Adduct $9 b$ (230 mg, $0.586 \mathrm{mmol})$ was dissolved in a MeOH/THF-mixture $(49 \mathrm{ml} / 15 \mathrm{ml})$ and cooled to $0{ }^{\circ} \mathrm{C}$. A second solution of $\mathrm{NaOH}(293 \mathrm{mg}, 7.325 \mathrm{mmol})$ in $30 \%$ aqueous $\mathrm{H}_{2} \mathrm{O}_{2}(15 \mathrm{ml})$ was prepared at $0{ }^{\circ} \mathrm{C}$ and dropped portion wise to the adduct solution. After stirring for 40 minutes at room temperature the reaction was stopped by diluting with $\mathrm{CH}_{2} \mathrm{Cl}_{2}$ and washed with ice-cold $\mathrm{Fe}(\mathrm{II}) \mathrm{SO}_{4}$-solution $(2 \mathrm{x})$. The combined aqueous phases were extracted three times with $\mathrm{CH}_{2} \mathrm{Cl}_{2}$ and the combined organic extracts were dried over $\mathrm{MgSO}_{4}$. After filtration and concentration in vacuo a flash chromatography on silica gel with petroleum ether/Et ${ }_{2} \mathrm{O}(1: 1)$ afforded $15(231 \mathrm{mg}, 97 \%)$ as a white foam. IR $\left(\mathrm{CHCl}_{3}, \mathrm{~cm}^{-1}\right): 2932$ (s), 2856 (m), 1716 (s), 1612 (w), 1516 (s), 1464 (w), 1444 (w), 1248 (s), 1180 (m); ${ }^{1} \mathrm{H}$ NMR (400 MHz, $\left.\mathrm{CDCl}_{3}\right): \delta=0.56(\mathrm{dbr}, 13 \mathrm{~Hz}, 1 \mathrm{H}) ; 0.75(\mathrm{~s}, 3 \mathrm{H}) ; 1.10-1.60(\mathrm{~m}, 4 \mathrm{H}) ; 1.67$ (dbr, $\left.13 \mathrm{~Hz}, 1 \mathrm{H}\right) ; 1.99$ (dtrbr, 9/4 Hz, 1H); 2.16 (dbr, $13 \mathrm{~Hz}) ; 2.69-2.98$ (m, 3H); 3.30 (d, $4 \mathrm{~Hz}, 1 \mathrm{H}) ; 3.49$ (dd, 4/1 Hz, $1 \mathrm{H}) ; 3.79$ (s, 3H); 3.89 (dd, 11/1 Hz, 1H); 5.29 (m, 1H); 5.33 (m, 1H); 5.93 (dd, 13/6 Hz, 1H); $5.97(\mathrm{~m}, 1 \mathrm{H}) ; 6.12(\mathrm{~d}, 6 \mathrm{~Hz}, 1 \mathrm{H}) ; 6.84(\mathrm{~d}, 10 \mathrm{~Hz}, 2 \mathrm{H}) ; 7.12$ (d, $10 \mathrm{~Hz}, 2 \mathrm{H})$; Exact mass Calcd.: 408.2101, found: 408.2106.

Diol adduct (16). Adduct 9c (1 g, $2.356 \mathrm{mmol})$ was dissolved in ethyl acetate $(20 \mathrm{ml})$ and acetonitrile $(20 \mathrm{ml})$. A solution of $\mathrm{RuCl}_{3} * 3 \mathrm{H}_{2} \mathrm{O}(35 \mathrm{mg}, 0.133 \mathrm{mmol})$ and $\mathrm{NaIO}_{4}(608 \mathrm{mg}$, $3.534 \mathrm{mmol})$ in water $(5 \mathrm{ml})$ was added at $0{ }^{\circ} \mathrm{C}$. After 40 minutes of stirring at room temperature, the reaction was quenched by addition of a saturated aqueous $\mathrm{Na}_{2} \mathrm{~S}_{2} \mathrm{O}_{3}$-solution $(24 \mathrm{ml})$. The mixture was stirred for another five minutes and extracted three times with EtOAc. Drying of the combined organic layers over $\mathrm{MgSO}_{4}$, filtration, evaporation of the solvent under reduced pressure and flash chromatography on silica gel with petroleum ether/ $\operatorname{Et}_{2} \mathrm{O}(1: 2)$ afforded 16 (770 mg, $71 \%$ ) as a white solid. IR $\left(\mathrm{CHCl}_{3}, \mathrm{~cm}^{-1}\right): 3572(\mathrm{w}), 3457(\mathrm{w}), 2932(\mathrm{~m}), 2856(\mathrm{w})$, 1732 (s), 1708 (s), 1612 (w), 1516 (s), 1440 (m), 1252 (s); ${ }^{1} \mathrm{H}$ NMR (200 MHz, $\left.\mathrm{CDCl}_{3}\right): \delta=0.53$ (dbr, $13 \mathrm{~Hz}, 1 \mathrm{H}) ; 0.80$ (s, 3H); 1.04-2.02 (m, 6H); 2.24 (dbr, $13 \mathrm{~Hz}, 1 \mathrm{H}) ; 2.60-3.23$ (m, 5H); 3.52 (sbr, 1H); 3.74 (s, 3H); 3.81 (s, 3H); 3.92 (dd, 8/4 Hz, 1H); 4.13 (dd, 3/2 Hz, 1H); 4.41 (dd, 2/4 $\mathrm{Hz}, 1 \mathrm{H}) ; 5.97$ (d, $6 \mathrm{~Hz}, 1 \mathrm{H}) ; 6.27(\mathrm{dd}, 6 / 13 \mathrm{~Hz}, 1 \mathrm{H}) ; 6.88$ (d, $9 \mathrm{~Hz}, 2 \mathrm{H}) ; 7.30$ (d, $9 \mathrm{~Hz}, 2 \mathrm{H})$; FAB-MS: $\mathrm{m} / \mathrm{z}(\%)=458\left(\mathrm{M}^{+}, 5\right), 240(100$, diene 1).

Lactone adduct (17). A solution of diol adduct $16(50 \mathrm{mg}, 0.109 \mathrm{mmol})$ in THF (2.5 ml) was treated with $6 \mathrm{~N} \mathrm{HCl}(1 \mathrm{ml})$. After 5 days of stirring at room temperature, the reaction was neutralized with $\mathrm{NaOH}(240 \mathrm{mg})$. The aqueous layer was extracted three times with $\mathrm{Et}_{2} \mathrm{O}$. After separation of the organic layer, drying over $\mathrm{MgSO}_{4}$, filtration and flash chromatography on silica gel with petroleum ether/ $\mathrm{Et}_{2} \mathrm{O}(1: 1) 17$ (10 $\left.\mathrm{mg}, 22 \%\right)$ was obtained as a white solid. IR $\left(\mathrm{CHCl}_{3}, \mathrm{~cm}^{-1}\right): 3587(\mathrm{w}), 3545$ (w), 2936 (m), 2860 (w), 1792 (m), 1760 (m), 1732 (m), 1612 
(w), 1516 (s), 1252 (s); ${ }^{1} \mathrm{H}$ NMR (400 MHz, $\left.\mathrm{CDCl}_{3}\right): \delta=0.48(\mathrm{dbr}, 13 \mathrm{~Hz}, 1 \mathrm{H}) ; 0.83$ (s, 3H); 1.16-2.25 (m, 7H); 2.96 (dd, 29/18 Hz, 1H); 3.26 (dd, 40/18 Hz, 1H); 3.57 (dd, 10/10 Hz, 1H); $4.04(\mathrm{~m}, 1 \mathrm{H}) ; 4.40(\mathrm{~m}, 2 \mathrm{H}) ; 5.75(\mathrm{~d}, 3 \mathrm{~Hz}, 1 \mathrm{H}) ; 6.09$ (dd, 9/6 Hz, 1H); 6.19 (d, $6 \mathrm{~Hz}, 1 \mathrm{H}) ; 6.89$ $(\mathrm{d}, 10 \mathrm{~Hz}, 2 \mathrm{H}) ; 7.20(\mathrm{~d}, 10 \mathrm{~Hz}, 2 \mathrm{H}) ;{ }^{13} \mathrm{C} \mathrm{NMR}\left(100 \mathrm{MHz}, \mathrm{CDCl}_{3}\right): \delta=15.02$ (p); $21.06(\mathrm{~s})$; $23.43(\mathrm{~s}) ; 26.68(\mathrm{~s}) ; 28.43(\mathrm{~s}) ; 45.33$ (s, doublet $27 \mathrm{~Hz}) ; 50.81$ (t, doublet $20 \mathrm{~Hz}) ; 54.64$ (p); $61.84(\mathrm{q}) ; 62.25$ (q); $75.32(\mathrm{t}) ; 86.38(\mathrm{t}$, doublet $28 \mathrm{~Hz}) ; 100.70$ (q, doublet $185 \mathrm{~Hz}) ; 2 \mathrm{x} 113.18$ (t); 2x 128.04 (t); 131.45 (q); 138.21 (t); 138.90 (t); 159.13 (q); 173.93 (q); 208.56 (q); FAB-MS: $\mathrm{m} / \mathrm{z}(\%)=426\left(\mathrm{M}^{+}, 6\right), 240(72$, diene 1$)$.

Ketone adduct (18). To a solution of adduct 9 a $(50 \mathrm{mg}, 0.136 \mathrm{mmol})$ in dry toluene $(2 \mathrm{ml}) \mathrm{L}$ Selectride $\left(1 \mathrm{M}\right.$ in THF) $(130 \mu \mathrm{l}, 0.136 \mathrm{mmol})$ was added at $-78{ }^{\circ} \mathrm{C}$. The reaction was stirred for 15 minutes followed by addition of a saturated aqueous $\mathrm{NH}_{4} \mathrm{Cl}$-solution. The suspension was warmed to room temperature and partitioned between water and $\mathrm{Et}_{2} \mathrm{O}$. The aqueous layer was separated, neutralized with $2 \mathrm{~N} \mathrm{HCl}$ and extracted three times with $\mathrm{Et}_{2} \mathrm{O}$. The combined organic extracts were washed with brine, dried over $\mathrm{MgSO}_{4}$ and filtered. Concentration in vacuo and a flash chromatography on silica gel with petroleum ether/Et ${ }_{2} \mathrm{O}(3: 1)$ afforded 18 (43 mg, 86 \%) as a white solid. IR $\left(\mathrm{CHCl}_{3}, \mathrm{~cm}^{-1}\right): 3000$ (m), 2936 (s), 2856 (m), 1704 (s), 1612 (w), 1516 (s), 1464 (w), 1444 (w), 1380 (w), 1248 (s); $\left.{ }^{1} \mathrm{H} \mathrm{NMR} \mathrm{(400} \mathrm{MHz,} \mathrm{CDCl}_{3}\right): \delta=0.50$ (dbr, $\left.13 \mathrm{~Hz}, 1 \mathrm{H}\right) ; 0.77$ (s, 3H); 1.08-2.31 (m, 11H); $2.63(\mathrm{dd}, 30 / 10 \mathrm{~Hz}, 1 \mathrm{H}) ; 3.62$ (d, $10 \mathrm{~Hz}, 1 \mathrm{H}) ; 3.79$ (s, 3H); 6.02 (dd, 10/6 Hz, 1H); 6.05 (d, $6 \mathrm{~Hz}, 1 \mathrm{H}) ; 6.86(\mathrm{~d}, 10 \mathrm{~Hz}, 2 \mathrm{H}) ; 7.18$ (d, $10 \mathrm{~Hz}, 2 \mathrm{H}) ;{ }^{13} \mathrm{C} \mathrm{NMR}(100 \mathrm{MHz}$, $\left.\mathrm{CDCl}_{3}\right): \delta=16.34(\mathrm{p}) ; 21.48(\mathrm{~s}) ; 23.52$ (s); 27.89 (s); 28.46 (p, doublet $\left.27 \mathrm{~Hz}\right) ; 28.54$ (s); 53.74 (t, doublet $19 \mathrm{~Hz}) ; 54.46$ (t); 55.32 (p); 60.01 (q); 61.14 (q); 66.01 (q); 94.06 (q, doublet $198 \mathrm{~Hz})$; 2x $113.34(\mathrm{t}) ; 2 \mathrm{x} 128.23(\mathrm{t}) ; 131.14(\mathrm{q}) ; 135.38(\mathrm{t}) ; 139.44(\mathrm{t}) ; 157.89(\mathrm{q}) ; 210.87(\mathrm{q})$; Exact mass Calcd:: 368.2152 , found: 368.2139 .

Cyclic ether (19). The reaction was performed according to the preparation of $\mathbf{1 8}$ with adduct 9a (50 mg, $0.14 \mathrm{mmol})$, L-Selectride (1 M in THF) $(301 \mu \mathrm{l}, 0.308 \mathrm{mmol})$, dry toluene $(2 \mathrm{ml})$ over a period of 15 minutes at $-78{ }^{\circ} \mathrm{C}$ followed by a flash chromatography on silica gel with petroleum ether/Et ${ }_{2} \mathrm{O}(3: 1) .19(39 \mathrm{mg}, 82 \%)$ was obtained as a white solid. IR $\left(\mathrm{CHCl}_{3}, \mathrm{~cm}^{-1}\right): 3000(\mathrm{~m})$, 2956 (s), 2928 (s), 2852 (m), 1612 (w), 1516 (s), 1464 (m), 1248 (s); ' H NMR (200 MHz, $\left.\mathrm{CDCl}_{3}\right): \delta=3.76(\mathrm{~s}, 3 \mathrm{H}) ; 3.81(\mathrm{~d}, 7 \mathrm{~Hz}, 3 \mathrm{H}) ; 1.00-2.20(\mathrm{~m}, 14 \mathrm{H}) ; 2.43(\mathrm{~d}, 2 \mathrm{~Hz}, 1 \mathrm{H}) ; 3.79(\mathrm{~s}$, $3 \mathrm{H}) ; 4.08(\mathrm{~m}, 1 \mathrm{H}) ; 4.70(\mathrm{~m}, 1 \mathrm{H}) ; 6.86(\mathrm{~d}, 10 \mathrm{~Hz}, 2 \mathrm{H}) ; 7.14(\mathrm{~d}, 10 \mathrm{~Hz}, 2 \mathrm{H}) ;{ }^{13} \mathrm{C} \mathrm{NMR}(100 \mathrm{MHz}$, $\left.\mathrm{CDCl}_{3}\right): \delta=16.52(\mathrm{p}) ; 18.20(\mathrm{p}) ; 21.33(\mathrm{~s}) ; 22.79(\mathrm{~s}) ; 25.64(\mathrm{t}) ; 25.99(\mathrm{~s}) ; 29.32$ (s); $31.38(\mathrm{t}) ;$ $31.82(\mathrm{~s}) ; 32.98(\mathrm{~s}) ; 33.37(\mathrm{q}) ; 41.26(\mathrm{q}) ; 51.23(\mathrm{t}) ; 55.20(\mathrm{p}) ; 74.68(\mathrm{t}) ; 85.69(\mathrm{t}) ; 2 \mathrm{x} 113.64(\mathrm{t}) ;$ 127.93 (q); 2x 129.54 (t); 158.33 (q); Exact mass Calcd.: 350.2246, found: 350.2246.

Alcohol adduct (20). Ketone adduct $18(50 \mathrm{mg}, 0.136 \mathrm{mmol})$ in dry toluene $(1 \mathrm{ml})$ was treated with K-Selectride $(298 \mu \mathrm{l}, 0.299 \mathrm{mmol})$ at $-78{ }^{\circ} \mathrm{C}$. The mixture was warmed to $0{ }^{\circ} \mathrm{C}$ and quenched with a $10 \%-\mathrm{NaOH}$-solution $(0.4 \mathrm{ml})$. After subsequent portion wise addition of a $30 \%$ aqueous $\mathrm{H}_{2} \mathrm{O}_{2}$-solution $(0.32 \mathrm{ml})$ stirring for three hours followed. The reaction mixture was extracted three times with $\mathrm{Et}_{2} \mathrm{O}$ and the combined organic layers were washed with a saturated aqueous $\mathrm{Na}_{2} \mathrm{~S}_{2} \mathrm{O}_{3}$-solution followed by treatment with brine. Evaporation of the solvent and flash chromatography on silica gel with petroleum ether/ $\mathrm{Et}_{2} \mathrm{O}$ (3:1) afforded 20 (32 mg, 
$64 \%$ ) as a white solid, mp $103.8^{\circ} \mathrm{C} . \mathrm{IR}\left(\mathrm{CHCl}_{3}, \mathrm{~cm}^{-1}\right): 3572(\mathrm{~m}), 3000(\mathrm{~m}), 2932(\mathrm{~s}), 2856(\mathrm{~m})$, 1612 (m), 1516 (s), 1244 (s), 1180 (s); ${ }^{1} \mathrm{H}$ NMR (200 MHz, $\left.\mathrm{CDCl}_{3}\right): \delta=0.47$ (dbr, $\left.13 \mathrm{~Hz}, 1 \mathrm{H}\right)$; 0.80 (s, 3H); 1.07-2.39 (m, 13H); 1.50 (d, $22 \mathrm{~Hz}, 3 \mathrm{H}) ; 3.15$ (dd, 10/6 Hz, 1H); 3.79 (s, $3 \mathrm{H}) ; 4.08$ (m, 1H); 5.97 (dd, 11/6 Hz, 1H); 6.20 (dd, 6/2 Hz, 1H); 6.88 (d, $10 \mathrm{~Hz}, 2 \mathrm{H}), 7.25$ (d, $10 \mathrm{~Hz}, 2 \mathrm{H})$; ${ }^{13} \mathrm{C} \mathrm{NMR}\left(100 \mathrm{MHz}, \mathrm{CDCl}_{3}\right): \delta=16.9$ (p); 21.1 (s); 23.9 (s); $28.0(\mathrm{~s}) ; 29.1$ (p); 33.2 (s); $47.8(\mathrm{t}) ;$ $53.0(\mathrm{t}) ; 55.1(\mathrm{p}) ; 60.6(\mathrm{q}) ; 62.1(\mathrm{q}) ; 65.5(\mathrm{q}) ; 65.9(\mathrm{t}) ; 97.1(\mathrm{q}) ; 2 \mathrm{x} 113.6(\mathrm{t}) ; 2 \mathrm{x} 128.2(\mathrm{t}) ; 131.1$ $(\mathrm{q}) ; 135.2(\mathrm{t}) ; 137.1(\mathrm{t}) ; 158.0(\mathrm{q})$; Exact mass Calcd.: 370.2308 , found: 370.2305 .

Cyclic ether (23). Adduct 9a (300 mg, $0.819 \mathrm{mmol})$ was dissolved in dry THF (26 ml). At room

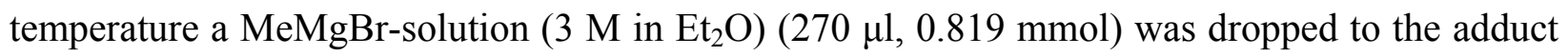
solution. The reaction was stopped after 5 minutes by addition of a saturated aqueous $\mathrm{NH}_{4} \mathrm{Cl}$ solution. After diluting the suspension with water and $\mathrm{Et}_{2} \mathrm{O}$ the aqueous layer was separated and extracted three times with $\mathrm{Et}_{2} \mathrm{O}$. The combined organic extracts were dried over $\mathrm{MgSO}_{4}$, filtered, and the solvent was evaporated under reduced pressure. Purification by flash chromatography on silica gel with petroleum ether/ $\mathrm{Et}_{2} \mathrm{O}(10: 1)$ afforded $23(212 \mathrm{mg}, 68 \%)$ as a colorless oil. IR $\left(\mathrm{CHCl}_{3}, \mathrm{~cm}^{-1}\right): 3000$ (m), 2964 (s), 2936 (s), 2864 (s), 1612 (w), 1512 (s), 1464 (w), 1444 (w), $1380(\mathrm{~m}), 1252$ (s), $1180(\mathrm{~m}), 1036(\mathrm{~m}) ;{ }^{1} \mathrm{H} \mathrm{NMR}\left(400 \mathrm{MHz}, \mathrm{CDCl}_{3}\right): \delta=0.76(\mathrm{~s}, 3 \mathrm{H}) ; 1.20(\mathrm{~s}$, $3 \mathrm{H}) ; 1.22-1.63(\mathrm{~m}, 8 \mathrm{H}) ; 1.79$ (d, $2 \mathrm{~Hz}, 3 \mathrm{H}) ; 1.94(\mathrm{~d}, 6 \mathrm{~Hz}, 1 \mathrm{H}) ; 2.04(\mathrm{~m}, 1 \mathrm{H}) ; 2.23(\mathrm{~m}, 1 \mathrm{H}) ; 2.40$ $(\mathrm{d}, 6 \mathrm{~Hz}, 1 \mathrm{H}) ; 3.79(\mathrm{~s}, 3 \mathrm{H}) ; 5.01(\mathrm{dd}, 5 / 1 \mathrm{~Hz}, 1 \mathrm{H}) ; 5.76(\mathrm{~m}, 1 \mathrm{H}) ; 6.85(\mathrm{~d}, 10 \mathrm{~Hz}, 2 \mathrm{H}) ; 7.22$ (d, 10 $\mathrm{Hz}, 2 \mathrm{H}) ;{ }^{13} \mathrm{C}$ NMR (100 MHz, $\left.\mathrm{CDCl}_{3}\right): \delta=18.49$ (p); 18.61 (p); 21.48 (s); 21.65 (s); 22.19 (p); $24.78(\mathrm{~s}) ; 34.28(\mathrm{~s}) ; 55.12(\mathrm{p}) ; 42.02(\mathrm{t}) ; 46.96(\mathrm{q}) ; 49.83(\mathrm{t}) ; 49.90(\mathrm{t}) ; 50.09(\mathrm{t}) ; 57.53(\mathrm{q})$; $73.71(\mathrm{q}) ; 86.89(\mathrm{t}) ; 90.29(\mathrm{q}) ; 2 \mathrm{x} 113.41(\mathrm{t}) ; 119.11(\mathrm{t}) ; 2 \mathrm{x} 128.19(\mathrm{t}) ; 132.55(\mathrm{q}) ; 136.70(\mathrm{q})$; 157.84 (q); Exact mass Calcd.: 362.2246, found: 362.2243.

Cyclic ether (24). To adduct 9 f $(52 \mathrm{mg}, 0.127 \mathrm{mmol})$ in dry THF (1 ml) was added a suspension of LAH (100 mg, $2.54 \mathrm{mmol})$ in THF $(1 \mathrm{ml})$. The mixture was refluxed over night before being quenched with a $0,1 \mathrm{~N} \mathrm{NaOH}$-solution at $0{ }^{\circ} \mathrm{C}$. The volume was increased with water and the mixture was dissolved with an excess of $2 \mathrm{~N}$ citric acid. The aqueous layers were extracted twice with $\mathrm{Et}_{2} \mathrm{O}$ and twice with $\mathrm{CH}_{2} \mathrm{Cl}_{2}$. After drying the combined organic extracts with brine and $\mathrm{MgSO}_{4}$, a flash chromatography on silica gel with petroleum ether/Et ${ }_{2} \mathrm{O}$ (5:1) afforded 24 (20 mg, $38 \%$ ) as a colorless oil. IR $\left(\mathrm{CHCl}_{3}, \mathrm{~cm}^{-1}\right): 3000$ (m), 2964 (s), 2948 (s), 2864 (m), 1612 (w), 1512 (s), 1464 (w), 1252 (s), 1180 (m), 1036 (m); ${ }^{1} \mathrm{H}$ NMR (200 MHz, $\left.\mathrm{CDCl}_{3}\right): \delta=0.85$ (s, $3 \mathrm{H}) ; 1.04$ (s, 9H); 1.10-1.66 (m, 8H); 1.98 (trbr, $5 \mathrm{~Hz}, 1 \mathrm{H}) ; 2.25$ (trbr, $6 \mathrm{~Hz}, 1 \mathrm{H}) ; 2.58$ (dd, 6/2 $\mathrm{Hz}, 1 \mathrm{H}) ; 2.77$ (dbr, $6 \mathrm{~Hz}, 1 \mathrm{H}) ; 3.79$ (s, 3H); 4.00 (m, 1H); 5.14 (dbr, $5 \mathrm{~Hz}, 1 \mathrm{H}) ; 5.74$ (dd, 7/2 Hz, 2H); $6.86(\mathrm{~d}, 9 \mathrm{~Hz}, 2 \mathrm{H}) ; 7.25$ (d, $9 \mathrm{~Hz}, 2 \mathrm{H})$; FAB-MS: m/z (\%) = $390\left(\mathrm{M}^{+}, 91\right)$.

Homoallylic alcohol adduct (26). Adduct 9a (500 mg, $1.357 \mathrm{mmol})$ was dissolved in dry toluene $(15 \mathrm{ml})$ and treated with DIBAL $\left(1,2 \mathrm{M}\right.$ in toluene) $(2.488 \mathrm{ml}, 2.985 \mathrm{mmol})$ at $-78{ }^{\circ} \mathrm{C}$. The reaction was warmed to room temperature and a saturated aqueous $\mathrm{NH}_{4} \mathrm{Cl}$-solution was added. The mixture was treated with a $\mathrm{K}_{2} \mathrm{CO}_{3}$-methanol paste and allowed to stir for an additional 5 minutes. The suspension was partitioned between water and $\mathrm{Et}_{2} \mathrm{O}$ then the aqueous layer was separated and extracted three times with $\mathrm{Et}_{2} \mathrm{O}$. Subsequent drying of the combined organic layers over $\mathrm{MgSO}_{4}$, filtration and concentration of the solution with the rotary evaporator 
provided the crude product. Purification by flash chromatography on silica gel with petroleum ether/ $\mathrm{Et}_{2} \mathrm{O}(3: 1)$ afforded 26 (382 $\mathrm{mg}, 80 \%$ ) as a white foam. IR $\left(\mathrm{CHCl}_{3}, \mathrm{~cm}^{-1}\right): 3584(\mathrm{~m}), 3000$ (m), 2928 (s), 2856 (m), 1612 (w), 1512 (s), 1252 (s), 1180 (s); ${ }^{1} \mathrm{H}$ NMR $\left(200 \mathrm{MHz}, \mathrm{CDCl}_{3}\right): \delta=$ 0.34 (dbr, $13 \mathrm{~Hz}, 1 \mathrm{H}) ; 0.86$ (s, 3H); 1.05-2.20 (m, 10H); 1.80 (sbr, 3H); 2.62 (dbr, $9 \mathrm{~Hz}, 1 \mathrm{H})$; $3.56(\mathrm{dd}, 9 / 7 \mathrm{~Hz}, 1 \mathrm{H}) ; 3.80(\mathrm{~s}, 3 \mathrm{H}) ; 4.11(\mathrm{~m}, 1 \mathrm{H}) ; 5.30(\mathrm{~m}, 1 \mathrm{H}) ; 5.94(\mathrm{~d}, 6 \mathrm{~Hz}, 1 \mathrm{H}) ; 6.23(\mathrm{~d}$, $6 \mathrm{~Hz}, 1 \mathrm{H}) ; 6.88(\mathrm{~d}, 9 \mathrm{~Hz}, 2 \mathrm{H}) ; 7.34(\mathrm{~d}, 9 \mathrm{~Hz}, 2 \mathrm{H}) ;{ }^{13} \mathrm{C} \mathrm{NMR}\left(100 \mathrm{MHz}, \mathrm{CDCl}_{3}\right): \delta=14.95(\mathrm{p}) ;$ 21.02 (s); 23.60 (s); 24.34 (p); 27.67 (s); 27.89 (s); 33.22 (s); $47.03(\mathrm{t}) ; 50.37(\mathrm{t}) ; 55.12(\mathrm{p}) ; 59.70$ (q); $62.00(\mathrm{q}) ; 66.03(\mathrm{q}) ; 69.55(\mathrm{t}) ; 2 \mathrm{x} 113.46(\mathrm{t}) ; 120.38(\mathrm{t}) ; 2 \mathrm{x} 128.19(\mathrm{t}) ; 129.40(\mathrm{q}) ; 131.63$ (q); $137.12(\mathrm{t}) ; 137.31(\mathrm{t}) ; 158.00(\mathrm{q}) ;$ FAB-MS: $\mathrm{m} / \mathrm{z}(\%)=350\left(\mathrm{M}^{+}, 4\right), 240(100$, diene 1).

(3aS,7R,7aR)-7-Fluoro-2,2,7-trimethyl-7,7a-dihydro-1,3-benzodioxol-4(3aH)-one (27). Acetonide adduct 14a $(298 \mathrm{mg}, 0.676 \mathrm{mmol})$ was pyrolized at $2 * 10^{-2}$ mbar and $350{ }^{\circ} \mathrm{C}$. The educt evaporated between $165{ }^{\circ} \mathrm{C}$ and $220{ }^{\circ} \mathrm{C}$ from the flask into the pyrolisation-tube. The crude product was collected in a flask behind the pyrolisation-tube cooled with liquid nitrogen. The flash chromatographic purification on silica gel with petroleum ether/ $\mathrm{Et}_{2} \mathrm{O}(5: 1)$ gave 27 (103 mg, $76 \%$ ) as a yellow oil. IR $\left(\mathrm{CHCl}_{3}, \mathrm{~cm}^{-1}\right): 2988(\mathrm{~m}), 2936(\mathrm{w}), 2896(\mathrm{w}), 1696(\mathrm{~s}), 1452$ (w), 1376 (m), 1224 (s), 1080 (s); ${ }^{1} \mathrm{H}$ NMR (400 MHz, $\left.\mathrm{CDCl}_{3}\right): \delta=1.38$ (s, 3H); 1.43 (s, 3H); 1.65 (d, $25 \mathrm{~Hz}, 3 \mathrm{H}) ; 4.51$ (d, $5 \mathrm{~Hz}, 1 \mathrm{H}) ; 4.55$ (ddd, 12/6/2 Hz, 1H); 6.16 (dd, 11/4 Hz, 1H); 6.75 (ddd, 11/5/2 Hz, 1H); ${ }^{13} \mathrm{C}$ NMR (100 MHz, $\mathrm{CDCl}_{3}$ ): $\delta=23.98$ (p, doublet $22 \mathrm{~Hz}$ ); $25.71(\mathrm{p}$ ); 27.16 (p); 74.24 (t); 78.36 (t, doublet $37 \mathrm{~Hz}) ; 89.63$ (q, doublet $161 \mathrm{~Hz}) ; 110.86(\mathrm{q}) ; 129.72$ (t); 146.06 (t); 194.78 (q); Exact mass Calcd.: 200.0849, found: 200.0843.

Methyl [(3aR,4R,7aS)-4-fluoro-2,2-dimethyl-7-oxo-3a,4,7,7a-tetrahydro-1,3-benzodioxol-4yl]acetate (28). The reaction was performed according to the preparation of 27 with acetonide adduct $14 \mathbf{b}(250 \mathrm{mg}, 0.501 \mathrm{mmol})$ at an evaporation temperature of approx. $150-240{ }^{\circ} \mathrm{C}$ and a flash chromatography on silica gel with petroleum ether/ $\mathrm{Et}_{2} \mathrm{O}(5: 1) .28(89 \mathrm{mg}, 69 \%)$ was obtained as a yellow oil. IR $\left(\mathrm{CHCl}_{3}, \mathrm{~cm}^{-1}\right): 2992(\mathrm{~m}), 2952$ (w), 1740 (s), 1700 (s), 1436 (m), $1376(\mathrm{~m}), 1228(\mathrm{~s}) ;{ }^{1} \mathrm{H}$ NMR $\left(400 \mathrm{MHz}, \mathrm{CDCl}_{3}\right): \delta=1.37(\mathrm{~s}, 3 \mathrm{H}) ; 1.43(\mathrm{~s}, 3 \mathrm{H}) ; 2.39$ (dd, 25/16 Hz, 1H); 2.58 (dd, 18/16 Hz, 1H); 3.76 (s, 3H); 4.02 (d, $6 \mathrm{~Hz}, 1 \mathrm{H}) ; 4.27$ (ddd, 13/6/2 Hz, 1H); $6.24(\mathrm{dd}, 11 / 3 \mathrm{~Hz}, 1 \mathrm{H}) ; 7.06(\mathrm{ddd}, 11 / 4 / 2 \mathrm{~Hz}, 1 \mathrm{H}) ;{ }^{13} \mathrm{C} \mathrm{NMR}\left(100 \mathrm{MHz}, \mathrm{CDCl}_{3}\right): \delta=25.61$ (p); 27.04 (p); 41.25 (s, doublet $25 \mathrm{~Hz}$ ); 52.04 (p); 74.13 (t); 77.37 (t, doublet $37 \mathrm{~Hz}$ ); 88.65 (q, doublet $167 \mathrm{~Hz}) ; 111.07$ (q); 130.42 (t); 143.45 (t); 168.49 (q); 194.46 (q); Exact mass Calcd.: 258.0904, found: 258.0909 .

(1S,5R,6R)-5-Allyl-5-fluoro-7-oxabicyclo[4.1.0]hept-3-en-2-one (29). The reaction was performed according to the preparation of 27 with epoxide adduct $15(216 \mathrm{mg}, 0.529 \mathrm{mmol})$ at an evaporation temperature of approx. $150-250^{\circ} \mathrm{C}$ and a flash chromatography on silica gel with petroleum ether/Et $2 \mathrm{O}(30: 1) .29(62 \mathrm{mg}, 70 \%)$ was obtained as a yellow oil. IR $\left(\mathrm{CHCl}_{3}, \mathrm{~cm}^{-1}\right)$ : 3084 (w), 3028 (w), 2984 (w), 2936 (w), 2912 (w), 1696 (s), 1640 (w), 996 (m); ${ }^{1} \mathrm{H}$ NMR $\left(200 \mathrm{MHz}, \mathrm{CDCl}_{3}\right): \delta=2.25(\mathrm{~m}, 2 \mathrm{H}) ; 3.01(\mathrm{dd}, 4 / 2 \mathrm{~Hz}, 1 \mathrm{H}) ; 3.08(\mathrm{~m}, 1 \mathrm{H}) ; 4.98(\mathrm{~m}, 2 \mathrm{H}) ; 5.97$ $(\mathrm{m}, 1 \mathrm{H}) ; 6.05(\mathrm{~m}, 1 \mathrm{H}) ; 6.51(\mathrm{~m}, 1 \mathrm{H}) ;{ }^{13} \mathrm{C} \mathrm{NMR}\left(100 \mathrm{MHz}, \mathrm{CDCl}_{3}\right): \delta=41.48(\mathrm{~s}) ; 52.54(\mathrm{t}) ;$ $56.65(\mathrm{t}$, doublet $43 \mathrm{~Hz}) ; 87.94$ (q, doublet $168 \mathrm{~Hz}) ; 121.08(\mathrm{~s}) ; 128.27(\mathrm{t}) ; 129.45(\mathrm{t}) ; 141.58(\mathrm{t})$; 
192.14 (q); MS (RT): m/z (\%) = $168\left(\mathrm{M}^{+}, 2\right), 151$ (7), 145 (18), 139 (32), 127 (56), 119 (28), 112 (35), 99 (100).

Methyl (3aR,7aR)-3a-fluoro-6-oxo-2,3,3a,6,7,7a-hexahydro-1H-indole-1-carboxylate (30). The reaction was performed according to the preparation of 27 with hydroindole adduct 12 (133 mg, $0.293 \mathrm{mmol}$ ) at an evaporation temperature of approx. 150-200 ${ }^{\circ} \mathrm{C}$ and a flash chromatography on silica gel with petroleum ether/ $\mathrm{Et}_{2} \mathrm{O}(1: 3) .30$ (63 mg, $100 \%$ ) was obtained as a colorless oil. IR $\left(\mathrm{CHCl}_{3}, \mathrm{~cm}^{-1}\right): 3000$ (m), 2956 (m), 2900 (w), 1692 (s), 1452 (s), 1392 (s), 1356 (m), 1072 (s); ${ }^{1} \mathrm{H}$ NMR (400 MHz, $\left.\mathrm{CDCl}_{3}\right): \delta=2.26(\mathrm{~m}, 2 \mathrm{H}) ; 2.45(\mathrm{~m}, 1 \mathrm{H}) ; 3.13$ (dd, 17/6 $\mathrm{Hz}, 1 \mathrm{H}) ; 3.64$ (m, 2H); 3.73 (s, 3H); 4.42 (m, 1H); 6.10 (d, $10 \mathrm{~Hz}, 1 \mathrm{H}) ; 6.89$ (dd, 10/4 Hz, 1H); ${ }^{13} \mathrm{C}$ NMR (100 MHz, $\left.\mathrm{CDCl}_{3}\right): \delta=33.77$ (s); 34.74 (s); 42.24 (s); 44.93 (s); 52.79 (p); 62.18 (t, doublet $23 \mathrm{~Hz}$ ); 96.72 (q, doublet $83 \mathrm{~Hz}) ; 130.67$ (t); 143.56 (t); 155.10 (q); 195.33 (q); Exact mass Calcd.: 213.0801, found: 213.0793.

\section{Acknowledgements}

Constant support of these investigations by the Deutsche Forschungsgemeinschaft and the Fonds der Chemischen Industrie is gratefully acknowledged.

\section{References}

1. Winterfeldt, E.; Beckmann, M.; Meyer, T.; Schulz, F. Chem. Ber. 1994, 127, 2505.

2. Zwanenburg, B.; Klunder, A. J. H.; Zhu, J. Chem. Rev. 1999, 99, 1163.

3. Wolter, M.; Borm, C.; Merten, E.; Wartchow, R.; Winterfeldt, E. Eur. J. Org. Chem. 2001, 4051.

4. Winterfeldt, E. Adv. in Asymm. Synth.; JAI Press Inc., 1997; Vol. 2, p 1.

5. Weinmann, H.; Winterfeldt, E. Synthesis 1996, 3, 357.

6. Beil, W.; Jones, P. G.; Nerenz, F.; Winterfeldt, E. Tetrahedron 1998, 54, 7273.

7. Tran-Hun-Dau, M. E.; Wartchow, R.; Winterfeldt, E.; Wong, Y. S. Chem. Eur. J. 2001, 7, 2349.

8. Bohlmann, R. Nachr. Chem. Tech. Lab. 1990, 38, 40.

9. Hagan, D. O.; Rzepa, H. S. Chem. Comm. 1997, 645.

10. Welch, J. T. Tetrahedron 1987, 43, 3123.

11. Iseki, K.; Kobayashi, Y. Rev. on Heteroatom Chem. 1995, 12, 211

12. (a) Buzetti, F.; Gäumann, E.; Hütter, R.; Keller-Schierlein, W.; Neipp, L.; Prelog, V., Zähner, H. Pharm. Acta. Helv. 1963, 38, 871. (b) Hara, M.; Akasaka, K.; Okabe, M.; Nakano, H.; Gomez, R.; Wood, D.; Tamanoi, F. Proc. Nat. Acad. Sci. USA 1993, 90, 2281. (c) Sattler, I.; Röhne, C.; Zeek, A. J. Org. Chem. 1993, 58, 6583. (d) König, G. M.; Wright, A. D. Heterocycles 1993, 36, 1351. (e) Goldenstein, K.; Fendert, T.; Proksch, P.; 
Winterfeldt, E Tetrahedron 2000, 56, 4173. (f) Ma, W.; Anderson, J. E.; McLaughlin, J. L. Heterocycles 1992, 34, 5. (g) Sandmeier, P.; Tamm, Ch. Helv. Chim. Acta 1989, 72, 784. (h) Bach, G.; Breiding-Mach, S.; Grabley, S.; Hamman, P.; Hütter, K.; Thierike, R.; Uhr, H.; Wink, J.; Zeek, A. Liebigs Ann. Chem. 1993, 241. (i) Carter, S. K.; Crooke, S. T. Mitomycin C, Current status and new developments; Academic Press: N.Y., 1979. (j) Wild, H. J. Org. Chem. 1993, 58, 7159. (k) Shiraga, Y.; Okano, K.; Akira, T.; Fukaya, C.; Yokoyama, K.; Tanaka, S.; Fukui, H.; Tabata, M. Tetrahedron 1988, 44, 4703. (1) Krohn, K.; Beckmann, K.; Flörke, U.; Aust, H. J.; Draeger, S.; Schulz, B.; Busemann, S.; Bringmann, G. Tetrahedron 1997, 53, 3101. (m) Perry, N. B.; Blunt, J. W.; McCombs, J. D.; Munro, M. H. G. J. Org. Chem. 1986, 51, 5476. (n) Golik, J.; Dubay, G.; Groenewold, G.; Kawaguchi, H.; Konishi, M.; Krishnan, B.; Ohkuma, H.; Saitoh, K.; Doyle, T. J. Am. Chem. Soc. 1987, 109, 3462. (o) Lee, M.; Dunne, T.; Siegel, M.; Chang, C.; Morton, G.; Borders, D. J. Am. Chem. Soc. 1987, 109, 3464.

13. Jacquesy, J. C.; Karam, O.; Jouannetaud, M. P. Tetrahedron Lett. 1994, 35, 2541.

14. Cason, J. Organic Reactions IV; J. Wiley Inc.; 1948, p 306.

15. Lettler, J. E. An Introduction to free radicals; Wiley: N.Y., 1993; p 58.

16. Sing, T. K. M.; Tai, V.; Tam, E. K. W. Angew. Chem. 1994, 106, 2408.

17. Jones, P. G.; Weinmann, H.; Winterfeldt, E. Angew. Chem. 1995, 107, 489.

18. (a) Nemoto, H.; Tanabe, T.; Fukumoto, K. J. Org. Chem. 1995, 60, 6785. (b) Cavazza, M.; Guella, G.; Nucci, L.; Pergola, F.; Biccherini, N.; Pietra, F. J. Chem. Soc., Perk. Trans. I 1993, 3117. (c) Kita, Y.; Tohma, H.; Kiguchi, K.; Anagaki, M.; Yakura, T. J. Org. Chem. 1991, 56, 435.

19. Seybold, G.; Jersak, U. Chem. Ber. 1977, 110, 1239. 